\title{
THE STELLAR POPULATIONS OF LOW-LUMINOSITY ACTIVE GALACTIC NUCLEI. II. SPACE TELESCOPE IMAGING SPECTROGRAPH OBSERVATIONS ${ }^{1}$
}

\author{
Rosa M. González Delgado, ${ }^{2}$ Roberto Cid Fernandes, ${ }^{3}$ Enrique Pérez, ${ }^{2}$ Lucimara P. Martins, ${ }^{4,5}$ \\ Thaisa Storchi-Bergmann, ${ }^{6}$ Henrique Schmitt, ${ }^{7,8}$ Timothy Heckman, ${ }^{9,10}$ and Claus Leitherer ${ }^{4}$ \\ Received 2003 July 2; accepted 2003 December 23
}

\begin{abstract}
We present a study of the stellar populations of low-luminosity active galactic nuclei (LLAGNs). Our goal is to search for spectroscopic signatures of young and intermediate-age stars and to investigate their relationship with the ionization mechanism in LLAGNs. The method used is based on the stellar population synthesis of the optical continuum of the innermost $(20-100 \mathrm{pc})$ regions in these galaxies. For this purpose, we have collected high spatial resolution optical (2900-5700 A) STIS spectra of 28 nearby LLAGNs that are available in the Hubble Space Telescope archive. The analysis of these data is compared with a similar analysis also presented here for 51 ground-based spectra of LLAGNs. Our main findings are as follows: (1) No features due to WolfRayet stars were convincingly detected in the STIS spectra. (2) Young stars contribute very little to the optical continuum in the ground-based aperture. However, the fraction of light provided by these stars is higher than $10 \%$ in most of the weak-[O I $([\mathrm{O} \mathrm{I}] / \mathrm{H} \alpha \leq 0.25)$ LLAGN STIS spectra. (3) Intermediate-age stars contribute significantly to the optical continuum of these nuclei. This population is more frequent in objects with weak than with strong [O I]. Weak-[O I] LLAGNs that have young stars stand out for their intermediate-age population. (4) Most of the strong-[O I] LLAGNs have predominantly old stellar population. A few of these objects also show a featureless continuum that contributes significantly to the optical continuum. These results suggest that young and intermediate-age stars do not play a significant role in the ionization of LLAGNs with strong [O I]. However, the ionization in weak-[O I] LLAGNs with young and/or intermediate-age populations could be due to stellar processes. A comparison of the properties of these objects with Seyfert 2 galaxies that harbor a nuclear starburst suggests that weak-[O I] LLAGNs are the lower luminosity counterparts of the Seyfert 2 composite nuclei.
\end{abstract}

Subject headings: galaxies: active — galaxies: nuclei — galaxies: starburst — galaxies: stellar content

On-line material: color figures

\section{INTRODUCTION}

Low-luminosity active galactic nuclei (LLAGNs) constitute a sizeable fraction of the nearby AGN population. These include low-luminosity Seyfert galaxies, low-ionization nuclear emission line regions (LINERs), and transition-type objects (TOs) whose properties are in between classical LINERs and $\mathrm{H}$ II nuclei. LLAGNs comprise $\sim \frac{1}{3}$ of all bright galaxies $\left(B_{T} \leq\right.$ 12.5 ) and are the most common type of AGNs (Ho, Filippenko, \& Sargent 1997a, hereafter HFS97).

\footnotetext{
${ }^{1}$ Based on observations with the NASA/ESA Hubble Space Telescope, obtained at the Space Telescope Science Institute, which is operated by the Association of Universities for Research in Astronomy, Inc., under NASA contract NAS 5-26555. Based on observations made with the Nordic Optical Telescope (NOT), operated on the island of La Palma jointly by Denmark, Finland, Iceland, Norway, and Sweden, in the Spanish Observatorio del Roque de los Muchachos of the Instituto de Astrofísica de Canarias.

${ }^{2}$ Instituto de Astrofísica de Andalucía (CSIC), P.O. Box 3004, 18080 Granada, Spain; rosa@iaa.es, eperez@iaa.es.

3 Departamento de Física-CFM, Universidade Federal de Santa Catarina, C.P. 476, 88040-900, Florianópolis, SC, Brazil; cid@astro.ufsc.br.

${ }^{4}$ Space Telescope Science Institute, 3700 San Martin Drive, Baltimore, MD 21218; martins@stsci.edu, leitherer@stsci.edu.

5 Instituto de Astronomía, Geofísica e Ciencias Atmosféricas, 05508-900 Sao Paulo, Brazil.

${ }^{6}$ Instituto de Física, Universidade Federal do Rio Grande do Sul, C.P. 15001, 91501-970, Poto Alegre, RS, Brazil; thaisa@if.ufrgs.br.

${ }^{7}$ National Radio Astronomy Observatory, 520 Edgemont Road, Charlottesville, VA 22903; hschmitt@nrao.edu.

8 Jansky Fellow.

9 Department of Physics and Astronomy, Johns Hopkins University, Baltimore, MD 21218; heckman@pha.jhu.edu.

${ }^{10}$ Also Adjunct Astronomer at STScI.
}

TOs could constitute rather mixed phenomena as suggested by the several excitation mechanisms that have been proposed to explain the origin of their energy source. Among these mechanisms are shocks, photoionization by a nonstellar $\mathrm{UV} / \mathrm{X}$-ray continuum (AGN), and photoionization by hot stars (see, e.g., review by Filippenko 1996). The possibility that some LINERs (as well as Seyfert galaxies) might be photoionized by hot stars has been suggested by several authors (e.g., Stasinska 1984; Filippenko \& Terlevich 1992; Shields 1992). These hot stars could be the product of the evolution of massive stars (the so-called warmers proposed by Terlevich \& Melnick 1985) or of intermediate-mass stars (the post-AGB stars investigated by Binette et al. 1994; Taniguchi, Shioya, \& Murayama 2000).

The massive star scenario has been reexamined by Barth \& Shields (2000), using detailed starburst plus photoionization modeling with evolutionary stellar synthesis models. These authors showed that the TO properties can be reproduced by short stellar bursts with ages of $\sim 3-6 \mathrm{Myr}$, when the ionizing continuum is dominated by emission from Wolf-Rayet (W-R) stars. More recent photoionization models with updated Starburst99 (Smith, Norris, \& Crowther 2002), which include new blanketed W-R and O atmospheres, are not able to reproduce the typical TO emission-line ratios (González Delgado et al. 2003).

There is further evidence in favor of the starburst scenario, coming from the detection of stellar wind lines in the ultraviolet spectra of some weak-[O I] LINERs and TOs (Maoz et al. 1998; Colina et al. 2002). In fact, similar spectroscopic features detected in Seyfert 2 galaxies are interpreted as due to a few 
million year old nuclear starbursts (Heckman et al. 1997; González Delgado et al. 1998).

About $20 \%$ of LLAGNs in the catalog of HFS97 required a broad component to fit the $\mathrm{H} \alpha$ emission (type 1 LINERs; Ho, Filippenko, \& Sargent 1997b). Some of these objects show double-peaked H lines (Storchi-Bergmann et al. 1997; Shields et al. 2000; Ho et al. 2000). In addition, recent X-ray observations of LLAGNs confirm that some objects are at the lowluminosity end of the AGN phenomenon and that they are powered by an accreting black hole (BH; Terasima, Ho, \& Ptak 2000; Ho et al. 2001).

Furthermore, recent high spatial resolution UV (Colina et al. 2002) and Chandra (Jiménez-Bailón et al. 2003) observations of the LLAGN NGC 4303 show that a super-stellar cluster (SSC) and a BH accreting with low radiative efficiency coexist within the inner few parsecs from the nucleus.

Considering the diversity of excitation mechanisms that can explain the emission-line spectrum of LLAGNs, we have started a project to examine the central stellar population in these objects, with the aim of finding clues about their physical origin and energy source. With this goal, we have carried out optical (3500-5500 $\AA$ ) spectroscopic observations of LLAGNs to (1) search for the presence of the broad W-R bump at the blue optical range (a blend of broad He II $\lambda 4686, \mathrm{~N}$ III $\lambda 4640$, and C IV 24650), (2) detect the absorption lines of He I and the highorder $\mathrm{H}$ I Balmer series lines (HOBLs), and (3) characterize their stellar populations. The W-R bump probes the presence of very young stars (few million years old), while $\mathrm{He}$ I and HOBLs probe the young (10-50 Myr) and intermediate-age (1001000 Myr) stars (González Delgado, Leitherer, \& Heckman 1999). In addition, this spectral range contains many metallic stellar lines typical of old and intermediate-age populations.

In a companion paper (Cid Fernandes et al. 2004, hereafter Paper I) we have presented the data and an empirical correlation between the stellar lines and the emission lines in LLAGNs, in particular the $[\mathrm{O} \mathrm{I}] / \mathrm{H} \alpha$ line ratio. Our main findings from Paper I are as follows: (1) No features due to W-R stars are convincingly detected, implying that massive stars contribute very little to the optical light. This happens even in the few cases where young stars are known to dominate the UV emission. (2) HOBLs, on the other hand, are detected in $40 \%$ of the sample. These lines are absent in most strong-[O I ] LLAGNs, but they are detected in about $50 \%$ of the weak-[O I] LLAGNs, defined as TOs and LINERs with $[\mathrm{O} \mathrm{I}] / \mathrm{H} \alpha \leq 0.25$. In fact, $\sim 90 \%$ of nuclei exhibiting HOBLs are weak-[O I] emitters.

The analysis in Paper I was carried out entirely in empirical terms, investigating connections between observed quantities. In this paper we present a stellar population synthesis analysis of the nuclear spectra of these objects. Because our results may be affected by the spatial resolution of the ground-based observations, we also present here the analysis of 28 LLAGNs observed at optical wavelengths with the Space Telescope Imaging Spectrograph (STIS) on board the Hubble Space Telescope (HST). The high spatial resolution provided by HST STIS may be crucial to spatially isolate the light from a central compact source or different circumnuclear star-forming knots from the underlying light emitted by the old stars in the inner bulge of these galaxies.

Another major goal of this study is to investigate the lowluminosity end of the "starburst-AGN connection." Powerful circumnuclear starbursts are present in $30 \%-50 \%$ of type 2 Seyfert galaxies (Cid Fernandes et al. 2001a and references therein). The statistics are not clear for type 1 Seyfert galaxies as a result of the difficulty in spotting circumnuclear starbursts against the bright nucleus (which is conveniently obscured in Seyfert 2 galaxies), but if unification is correct, the fraction of starburst plus AGN composites should be similar in Seyfert 1 and Seyfert 2 galaxies. If LINERs are just "mini-Seyferts," one would naively expect to find circumnuclear starbursts in about $30 \%-50 \%$ of them. Different incidence rates could be due to evolutionary effects, which, if present, should also be detected in a comparative stellar population analysis of Seyfert 2 galaxies and LINERs.

This paper is organized as follows: In $\S 2$ we describe the sample and the STIS observations. In $\S 3$ we present the STIS spectra and measurements of their properties. An empirical population synthesis (EPS) analysis of the whole sample (ground-based plus STIS) is presented in $\S 4$. The results of this analysis are discussed in $\S 5$, where we investigate the connection between the inferred stellar populations and emission-line properties, compare our results with those obtained for Seyfert 2 and starburst galaxies, and speculate on possible evolutionary scenarios. Finally, $\S 6$ summarizes our conclusions.

\section{OBSERVATIONS AND DATA REDUCTION}

\subsection{Galaxy Sample}

Our sample of LLAGNs was drawn entirely from the HFS97 catalog because it is the most complete and homogeneous survey of LINERs and TOs available for the local universe. In addition to the galaxies presented in Paper I, we have selected LLAGNs observed with STIS in the 2900-5700 $\AA$ spectral range. Spectra of 32 LLAGNs are available in the HST archive, of which 28 galaxies (17 TOs and 11 LINERs according to the classification of HFS97) are suitable for a stellar population analysis. Eight of these TOs have also been observed from the ground by us. These data are complemented with STIS spectra of four galaxies classified by HFS97 as H II nuclei, plus NGC 1023, a nonactive galaxy. Most of these observations are from proposals 8607 (PI: L. C. Ho) and 7361 (PI: H.-W. Rix). These projects include 24 nearby early-type ( $\mathrm{S} 0-\mathrm{Sb}$ ) galaxies and 15 TOs from the HFS97 catalog that are closer than $17 \mathrm{Mpc}$ and have emission lines with fluxes larger than $10^{-15} \mathrm{ergs} \mathrm{s}^{-1} \mathrm{~cm}^{-2} \AA^{-1}$ in a $2^{\prime \prime} \times 4^{\prime \prime}$ nuclear aperture. Observations of NGC 1023, NGC 3507, NGC 3998, and NGC 4261 are from proposals 7566 (PI: R. Green), 7357 (PI: L. C. Ho), 8839 (PI: L. Dressel), and 8236 (PI: S. Baum), respectively. Properties of these objects are listed in Table 1.

This collection plus data from Paper I increase the sample analyzed here to $33 \mathrm{TOs}$ and 40 LINERs, which represents $49 \%$ of the TOs and $42 \%$ of the LINERs in the HFS97 sample. If we adopt a slightly different classification criterion for LLAGNs, as we did in Paper I, placing the dividing line between the two subtypes at $\left[\mathrm{O}_{\mathrm{I}}\right] / \mathrm{H} \alpha=0.25$, our sample comprises 24 strong-[O I] and 47 weak-[O I] LLAGNs, corresponding to $43 \%$ and $44 \%$ of these types of galaxies in the HFS97 sample. Morphological type and distance distributions are presented and compared with the full HFS97 sample in Figure 1. Our sample has the same median morphological type as the HFS97 sample: S0 and Sab for strong- and weak-[O I] LLAGNs, respectively. With respect to the distance, the median in our sample is 17 and $23 \mathrm{Mpc}$ for weak- and strong[O I] LLAGNs, respectively. The corresponding values in the HFS97 sample are 20 and $22 \mathrm{Mpc}$. 
TABLE 1

LLAGNs with Archival STIS/CCD (G430L) Spectra

\begin{tabular}{|c|c|c|c|c|c|c|c|c|c|}
\hline $\begin{array}{l}\text { Name } \\
\text { (1) }\end{array}$ & $\begin{array}{l}\text { Type } \\
(2)\end{array}$ & $\begin{array}{c}\text { Morphology } \\
\text { (3) }\end{array}$ & $\begin{array}{c}v \\
\left(\mathrm{~km} \mathrm{~s}^{-1}\right) \\
(4)\end{array}$ & $\begin{array}{c}\text { Distance } \\
(\mathrm{Mpc}) \\
(5)\end{array}$ & $\begin{array}{c}1^{\prime \prime} \\
\left(\mathrm{pc}^{2}\right) \\
(6)\end{array}$ & $\begin{array}{c}{\left[\begin{array}{ll}\mathrm{O} & \mathrm{I}] / \mathrm{H} \alpha \\
\text { (7) }\end{array}\right.}\end{array}$ & $\begin{array}{l}H S T \text { ID } \\
\quad(8)\end{array}$ & $\begin{array}{c}\text { Exposure } \\
\text { (s) } \\
\text { (9) }\end{array}$ & $\begin{array}{l}\text { P.A. } \\
\text { (deg) } \\
(10)\end{array}$ \\
\hline NGC $2685^{*} \ldots \ldots \ldots$. & $\mathrm{S} 2 / \mathrm{T} 2$ & $\mathrm{~S}_{3}(7)$ pec & 869 & 16.2 & 78 & $0.13 b$ & 8607 & 2585 & 54.4 \\
\hline NGC $2787 \ldots \ldots \ldots \ldots . .$. & L1.9 & $\mathrm{SB}(\mathrm{r}) 0+$ & 691 & 13.0 & 63 & $0.55 b$ & 7361 & 1864 & 213.2 \\
\hline NGC $3368 \ldots \ldots \ldots . . .$. & L2 & $\mathrm{SAB}(\mathrm{rs}) \mathrm{ab}$ & 897 & 8.1 & 39 & 0.18 & 7361 & 1574 & 249.5 \\
\hline NGC $3489 \ldots \ldots \ldots . .$. & $\mathrm{T} 2$ & $\mathrm{SAB}(\mathrm{rs}) 0+$ & 701 & 6.4 & 31 & $0.11 \mathrm{~b}$ & 7361 & 1644 & 239.1 \\
\hline 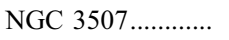 & L2 & $\mathrm{SB}(\mathrm{s}) \mathrm{b}$ & 978 & 19.8 & 96 & 0.18 & 7357 & 900 & 89.9 \\
\hline NGC $3627 * \ldots \ldots \ldots$. & $\mathrm{T} 2 / \mathrm{S} 2$ & S(s)bII.2 & 703 & 6.6 & 32 & 0.13 & 8607 & 2349 & 80.1 \\
\hline NGC $3675 \ldots \ldots \ldots \ldots . .$. & $\mathrm{T} 2$ & $\mathrm{SA}(\mathrm{s}) \mathrm{b}$ & 766 & 1.8 & 9 & $0.12 b$ & 8607 & 2472 & 205.9 \\
\hline NGC $3953 \ldots \ldots \ldots \ldots . . .$. & $\mathrm{T} 2$ & $\mathrm{SB}(\mathrm{r}) \mathrm{bc}$ & 1053 & 17 & 82 & $0.12 b$ & 8607 & 2561 & 79.1 \\
\hline 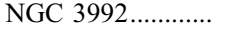 & T2: & $\mathrm{SB}(\mathrm{rs}) \mathrm{bc}$ & 1048 & 17 & 82 & $0.13 \mathrm{c}$ & 7361 & 1796 & 155.3 \\
\hline 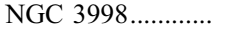 & L1.9 & SA0 & 1049 & 21.6 & 105 & 0.53 & 8839 & 2827 & 304.3 \\
\hline NGC $4143 \ldots \ldots \ldots \ldots$ & L1.9 & $\mathrm{SAB}(\mathrm{s}) 0$ & 783 & 17 & 82 & 0.71 & 7361 & 1707 & 139.0 \\
\hline NGC $4150 * \ldots \ldots \ldots .$. & $\mathrm{T} 2$ & $\mathrm{~S}_{3}(4) / \mathrm{a}$ & 244 & 9.7 & 47 & 0.13 & 8607 & 2395 & 240.1 \\
\hline NGC $4203 \ldots \ldots \ldots \ldots . .$. & L1.9 & SAB0- & 1085 & 9.7 & 47 & $1.22 \mathrm{~b}$ & 7361 & 1630 & 105.6 \\
\hline NGC $4261 \ldots \ldots \ldots . . .$. & L2 & $\mathrm{E} 2+$ & 2210 & 35.1 & 170 & 0.49 & 8236 & 1891 & 157.9 \\
\hline NGC $4314 \ldots \ldots \ldots . .$. & L2 & $\mathrm{SB}(\mathrm{rs}) \mathrm{a}$ & 962 & 9.7 & 47 & $0.18 \mathrm{~b}$ & 7361 & 1668 & 105.3 \\
\hline NGC $4321 \ldots \ldots \ldots \ldots . . .$. & $\mathrm{T} 2$ & $\mathrm{SAB}(\mathrm{s}) \mathrm{bc}$ & 1234 & 16.8 & 81 & 0.11 & 7361 & 1642 & 92.9 \\
\hline NGC $4414 \ldots \ldots \ldots \ldots . . .$. & T2: & $\mathrm{SA}(\mathrm{rs}) \mathrm{c}$ & 719 & 9.7 & 47 & $0.14 \mathrm{u}$ & 8607 & 2395 & 125.1 \\
\hline NGC 4429............... & $\mathrm{T} 2$ & $\mathrm{SA}(\mathrm{r}) 0+$ & 1137 & 16.8 & 81 & $0.097 \mathrm{u}$ & 8607 & 2349 & 81.1 \\
\hline NGC $4435 \ldots \ldots \ldots \ldots . . .$. & $\mathrm{T} 2$ & $\mathrm{SB}(\mathrm{s}) 0$ & 781 & 16.8 & 81 & $0.13 b$ & 7361 & 1644 & 89.6 \\
\hline NGC $4450 \ldots \ldots \ldots \ldots . .$. & L1.9 & $\mathrm{SA}(\mathrm{s}) \mathrm{ab}$ & 1956 & 16.8 & 81 & 0.67 & 7361 & 1669 & 233.0 \\
\hline NGC $4459 \ldots \ldots \ldots . .$. & $\mathrm{T} 2$ & $\mathrm{SA}(\mathrm{r}) 0+$ & 1202 & 16.8 & 81 & $0.13 \mathrm{u}$ & 7361 & 1644 & 92.9 \\
\hline NGC $4548 \ldots \ldots \ldots . . .$. & L2 & $\mathrm{SB}(\mathrm{rs}) \mathrm{b}$ & 485 & 16.8 & 81 & 0.23 & 7361 & 1644 & 73.2 \\
\hline NGC 4569*.......... & $\mathrm{T} 2$ & $\mathrm{SO}_{3}(3)$ & -311 & 16.8 & 81 & 0.062 & 8607 & 2349 & 100.0 \\
\hline NGC $4596 \ldots \ldots \ldots \ldots . . .$. & L2:: & $\mathrm{SB}(\mathrm{r}) 0+$ & 1874 & 16.8 & 81 & $0.27 \mathrm{u}$ & 7361 & 1671 & 250.3 \\
\hline NGC 4826*.......... & $\mathrm{T} 2$ & (R)SA(rs)ab & 411 & 4.1 & 20 & 0.073 & 8607 & 2356 & 88.1 \\
\hline NGC $5055^{*} \ldots \ldots \ldots .$. & $\mathrm{T} 2$ & S(s)bcII-III & 516 & 7.2 & 35 & $0.17 \mathrm{u}$ & 7361 & 1707 & 164.5 \\
\hline NGC 6503*.......... & $\mathrm{T} 2 / \mathrm{S} 2$ & $\mathrm{~S}(1) \mathrm{cII} .8$ & 26 & 6.1 & 30 & 0.08 & 8607 & 2687 & 315.1 \\
\hline NGC $7331 * \ldots \ldots \ldots .$. & $\mathrm{T} 2$ & $\mathrm{~S}(\mathrm{rs}) \mathrm{bI}-\mathrm{II}$ & 835 & 14.3 & 69 & $0.097 \mathrm{u}$ & 8607 & 2395 & 358.9 \\
\hline 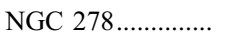 & $\mathrm{H}$ & $\mathrm{SAB}(\mathrm{rs}) \mathrm{b}$ & 639 & 11.8 & 57 & $0.022 b$ & 7361 & 1786 & 47.6 \\
\hline NGC $3351 \ldots \ldots \ldots \ldots . . .$. & $\mathrm{H}$ & $\mathrm{SB}(\mathrm{r}) \mathrm{b}$ & 778 & 8.1 & 39 & 0.019 & 7361 & 1671 & 245.6 \\
\hline NGC $4245 \ldots \ldots \ldots . . . .$. & $\mathrm{H}$ & $\mathrm{SB}(\mathrm{r}) 0 / \mathrm{a}$ & 890 & 9.7 & 47 & $0.038 \mathrm{u}$ & 7361 & 1668 & 85.7 \\
\hline NGC $4800 \ldots \ldots \ldots \ldots . . . . .$. & $\mathrm{H}$ & $\mathrm{SA}(\mathrm{rs}) \mathrm{b}$ & 808 & 15.2 & 74 & $0.041 \mathrm{~b}$ & 7361 & 1739 & 177.5 \\
\hline NGC $1023 * \ldots \ldots \ldots .$. & Abs & $\mathrm{SB}(\mathrm{rs}) 0$ & 632 & 10.5 & 51 & $\ldots$ & 7566 & 2475 & 93.1 \\
\hline
\end{tabular}

Notes.-Galaxies labeled with an asterisk have been observed also at NOT. Col. (1): Galaxy name. Col. (2): Spectral class (according to HFS97 criteria). Col. (3): Hubble type. Col. (4): Radial velocity. Col. (5): Distance. Col. (6): Angular scale. Col. (7): [O I]/H $\alpha$ flux ratio. Col. (8): HST proposal ID number. Col. (9): Exposure time. Col. (10): Slit P.A.

\subsection{Observations}

The observations were obtained with the STIS/CCD detector with a $52^{\prime \prime} \times 0.2$ slit (except for NGC 3507, which was observed with a 0.5 slit) and the G430L grating. The spectra cover the wavelength range $2900-5700 \AA$ with a dispersion of $2.7 \AA$ pixel $^{-1}$, giving a minimum FWHM spectral resolution of $\sim 4 \AA$ for point sources and up to $\sim 11 \AA$ for extended sources. After an initial acquisition exposure of a few seconds through the optical long-pass filter, the slit was placed across each nucleus at a randomly oriented position angle (P.A.). The CCD spatial scale is $0.05 \mathrm{pixel}^{-1}$. Observations from proposals 8607 and 8236 were binned every 2 pixels, yielding a spatial sampling of 0 "' 1 .

The data were calibrated with the standard STScI pipeline, meaning that the spectra were bias and dark subtracted, flatfielded, cleaned of cosmic rays, corrected for geometrical distortion, and flux-calibrated. The total integration time was split into two or more individual exposures. In some objects, the telescope was offset by several pixels along the slit direction between repeated exposures to aid in the removal of hot pixels. If there are more than two exposures of the same object, the median frame was obtained. For those galaxies with two individual exposures, we combined the two-dimensional spectra using a statistical differencing technique similar to that used by Pogge \& Martini (2002) for WFPC2 images. The technique consists of obtaining for each individual exposure a mask frame containing only the hot pixels and cosmic-ray hits, which is then subtracted from the original spectrum. The mask is obtained as follows. First, for each pair of exposures, a difference spectrum is formed by subtracting one spectrum in the pair from the other. The resulting frames consist mainly of positive and negative cosmic rays and hot pixels. A pair of mask frames is formed by separating the remaining positive and negative pixels, setting all the pixels within \pm a few $\sigma$ of the mean residual background level on the difference spectrum to zero. This technique works well when the pair of original spectra have similar spatial distributions along the slit and background.

All the spectra have been treated as extended continuum sources when converting from surface brightness units in the two-dimensional frames to flux units (ergs $\mathrm{s}^{-1} \mathrm{~cm}^{-2} \AA^{-1}$ ). Finally, the spectra have been corrected for redshift assuming the heliocentric radial velocities listed in Table 1. 

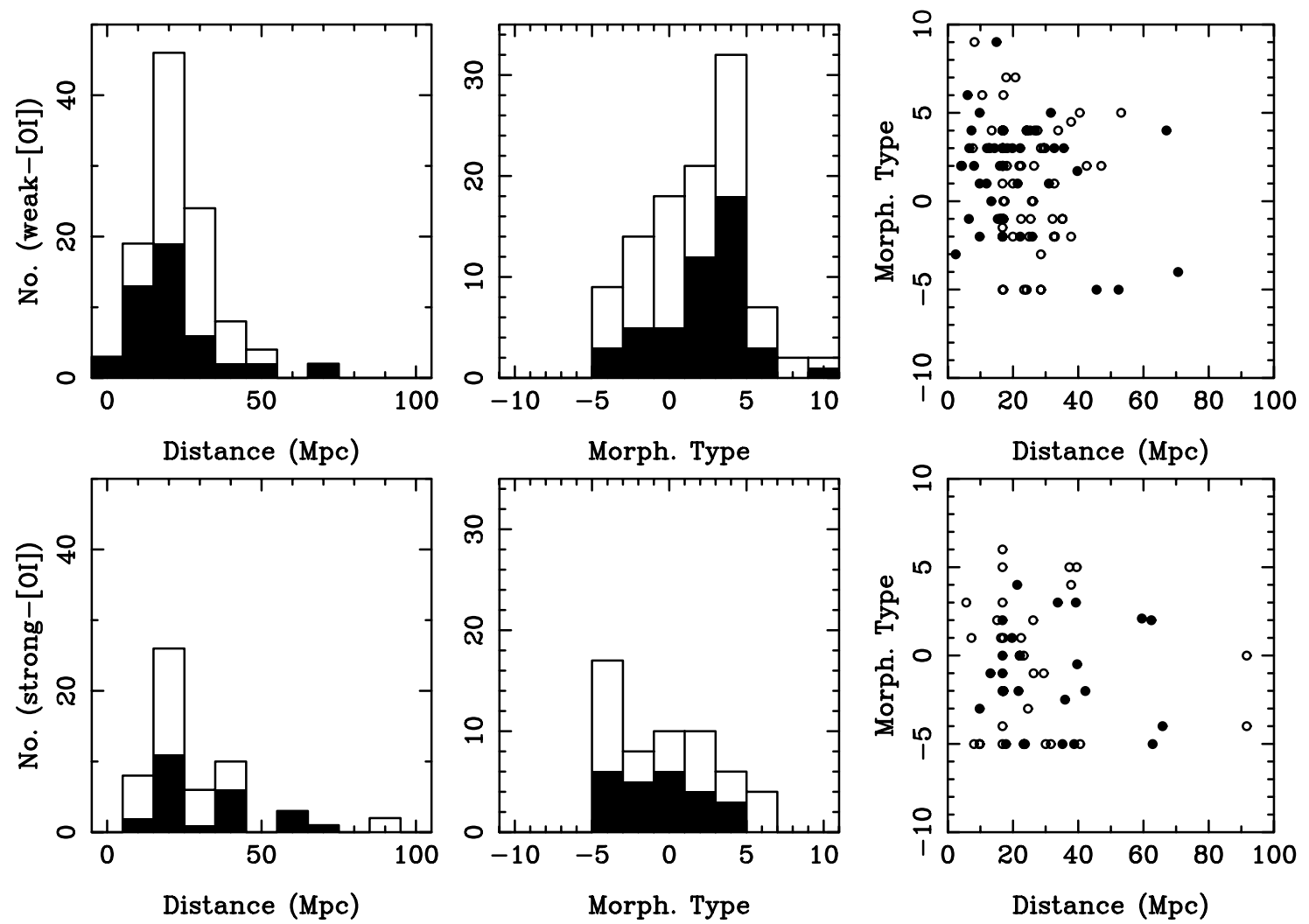

FIG. 1.-Distance and morphological type distributions for the strong-[O I] (bottom) and weak-[O I] (top) LLAGNs in the HFS97 sample. Filled areas in the histograms indicate the sample analyzed here.

\section{RESULTS}

\subsection{Central Morphology and Nuclear Spectral Extractions}

To extract the nuclear spectra of these galaxies, we have examined the spatial distribution of the continuum at $4700 \AA$ along the slit. In all the objects, the central surface brightness is extended, with a symmetric distribution about the maximum; in many of them, this indicates that the bulge component dominates the central continuum light. LLAGNs that have this type of distribution are NGC 2787, NGC 3489, NGC 3992, NGC 4429, NGC 4450, NGC 4596, and NGC 7331. A few of the galaxies show a very sharp distribution that contains most of the central flux. These are NGC 3998, NGC 4321, and NGC 4569. While the sharp brightness distribution in NGC 4321 and NGC 4569 could be produced by a nuclear stellar cluster, the origin in NGC 3998 is not clear. Other objects, like NGC 3627 , NGC 4150, NGC 4261, NGC 4435, NGC 4548, and NGC 6503, show a central complex structure that could be produced by the superposition of dust lanes and several clusters randomly distributed or in a circumnuclear ring. To better inspect the central morphology of these LLAGNs, we have retrieved the WFPC2 optical images available in the $H S T$ archive. These images confirm the morphology suggested by the spectral central surface brightness profile. A detailed analysis of the central morphology and its relation with the nuclear stellar population is being carried out (R. M. González Delgado 2004, in preparation). Figure 2 shows images and slit profiles of a few representative examples.

We have extracted two spectra for each galaxy (except in the case of NGC 4150, for which we have extracted three spectra; see Fig. 3) corresponding to the central 0.3 (called $b$ spectra) and $1^{\prime \prime}$ (called $a$ spectra). These two extractions allow us to check if there is a change of the stellar population on a spatial scale of a few tens of parsecs. A direct comparison of the spectra of these two extractions indicates that the dominant stellar population on scales of $1^{\prime \prime}$ is also the dominant stellar population on the $0 . " 3$ scale.

Eight TOs (NGC 2685, NGC 3627, NGC 4150, NGC 4569, NGC 4826, NGC 5055, NGC 6503, NGC 7331) and NGC 1023 have also been observed by us from the ground. We have compared the $a$ spectra with the nuclear spectra obtained from the ground. The strongest variations are found in NGC 4569 and NGC 4150, with a significant change in the shape of the continuum and/or the strength of the absorption lines. In the case of NGC 4569, the ground-based nuclear spectrum is somewhat redder and presents stronger metallic lines (such as the $\mathrm{G}$ band and $\mathrm{Ca}$ II $\mathrm{K}$ ) than the STIS $a$ spectrum, indicating that a larger fraction of the bulge population contributes to the ground-based extraction $\left(1^{\prime \prime} \times 1^{\prime \prime} 1\right)$ compared to the STIS extraction $\left(1^{\prime \prime} \times 00^{\prime \prime} 2\right)$. In the case of NGC 4150 the differences in the shape of the nuclear spectra can be associated with the dust lane structure that crosses the center and the different P.A. of the slit in the ground-based observations with respect to the STIS observations. In the remaining objects, the STIS and ground-based nuclear spectra are visually similar (see, e.g., NGC 3627 in Fig. 4 in this paper and Fig. 8 in Paper I), although some differences in the stellar populations are obtained through the EPS analysis discussed in $\S 4$.

\subsection{Empirical Stellar Population Classification}

In Paper I LLAGNs are classified empirically by comparing their spectra with those of nonactive galaxies dominated by stellar populations of different ages. The spectra were separated into four classes labeled by $\eta=Y, I, I / O$, and $O$ : 

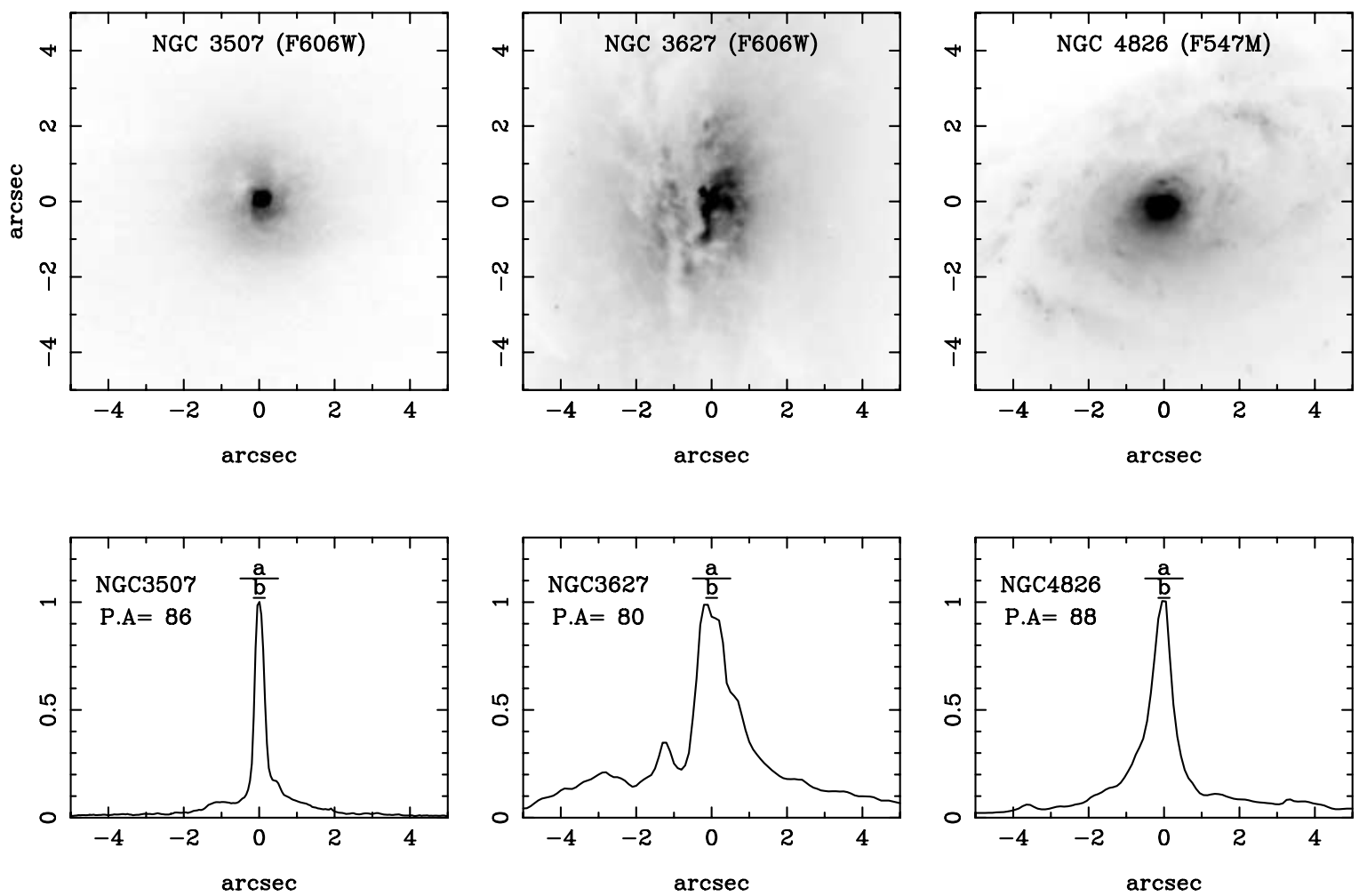

FIg. 2.-Top: WFPC2 images of NGC 3507, NGC 3627, and NGC 4826 through the filters F606W and F547M. North is up and east to the left. Bottom: Surface brightness profiles along the STIS slit of the optical continuum at $4700 \AA$. The extensions of the $a$ and $b$ spectral extractions are marked by horizontal lines and labeled.
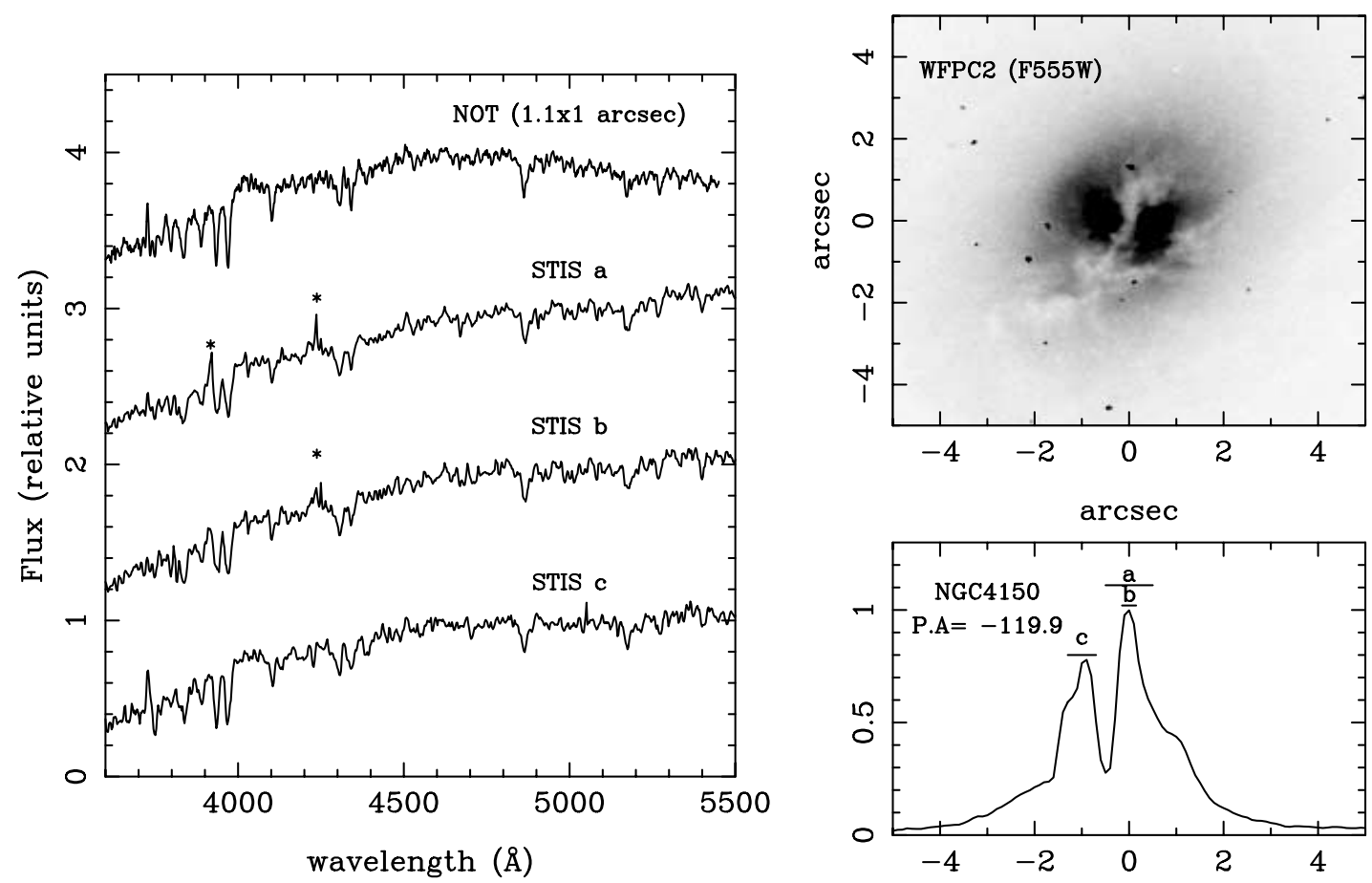

FIG. 3.-WFPC2 image through the F555W filter of NGC 4150 (top right), surface brightness profile along the STIS slit of the optical continuum at $4700 \AA$ (bottom right), and STIS spectra compared with the ground-based nuclear spectrum (left). The spectra are normalized to the flux at $4800 \AA$ and shifted vertically for clarity. 

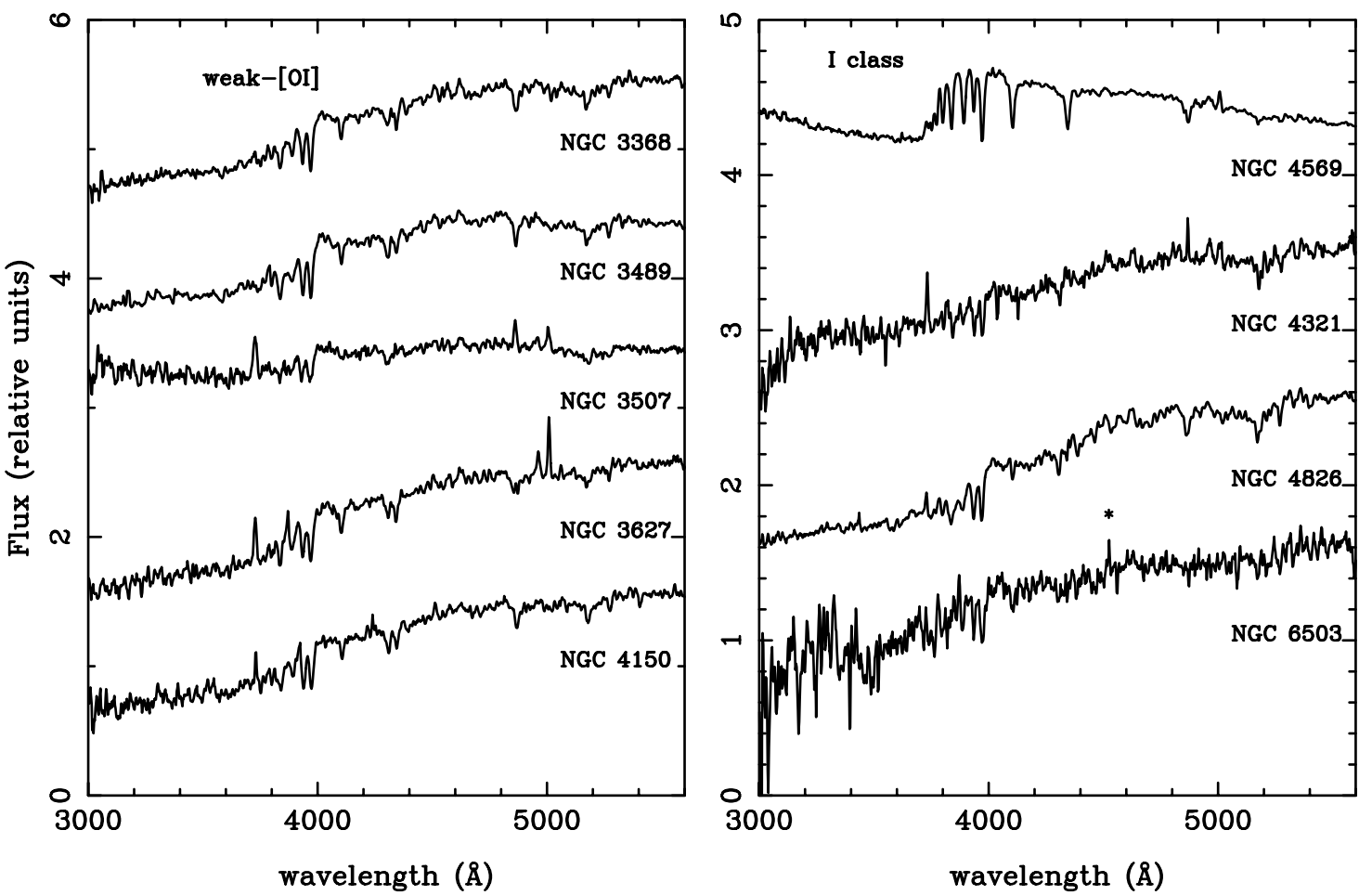

FIG. 4.-Nuclear spectra of weak-[O I] sources in the $I$ class, i.e., with an intermediate-age population

Y: Galaxies with young $\left(\leq 10^{7} \mathrm{yr}\right)$ stellar population, characterized by a blue continuum and very diluted metallic absorption lines.

I: Galaxies with a dominant intermediate-age population $\left(10^{8}-10^{9} \mathrm{yr}\right)$, characterized by prominent HOBLs in absorption.

$\mathrm{I} / \mathrm{O}$ : Galaxies with a mixture of intermediate-age and older stars. These galaxies do not have visible HOBLs in absorption, but the metallic lines are weaker than in an old stellar population.

$O$ : Galaxies dominated by an old $\left(10^{10} \mathrm{yr}\right)$ stellar population, with strong metallic lines, such as $\mathrm{Ca} \mathrm{K}$ and $\mathrm{H}, \mathrm{CN}, \mathrm{G}$ band, and $\mathrm{Mg}$ II.

Here we proceed in the same way, comparing the STIS spectra with the template galaxies observed from the ground. Figures 4-7 group the weak- and strong-[O I] LLAGN spectra in the $I, I / O$, and $O$ categories. As in Paper I, this classification is confirmed by modeling of the starlight by a combination of a base of five template galaxies representative of the $O$ (NGC 1023 and NGC 2950), I/O (NGC 221), I (NGC 205), and $Y$ (NGC 3367) classes. The results of this combination indicate that all the nuclei that belong to the $O$ have relative contribution at $4020 \AA$ of NGC 1023 plus NGC 2950 larger than $75 \%$, and those that belong to the $I$ class have relative contribution at $4020 \AA$ of NGC 205 larger than $30 \%$. These results are in perfect agreement with those obtained from the modeling of the stellar light using the population synthesis technique presented in $\S 4$.

As in the ground-based spectra, none of the STIS LLAGNs resemble pure young stellar systems. No features due to W-R stars were detected directly in the STIS spectra of either LINERs or TOs, in neither extraction $b\left(0,3 \times 00^{\prime \prime} 2\right)$ nor $a$ $\left(1^{\prime \prime} \times 0.2\right)$. If W-R stars are present in the nuclei of some LLAGNs, they must contribute very little to the optical light, given that even observing through a very narrow slit, thus minimizing the bulge light, the broad bump at $4660 \AA$ is not

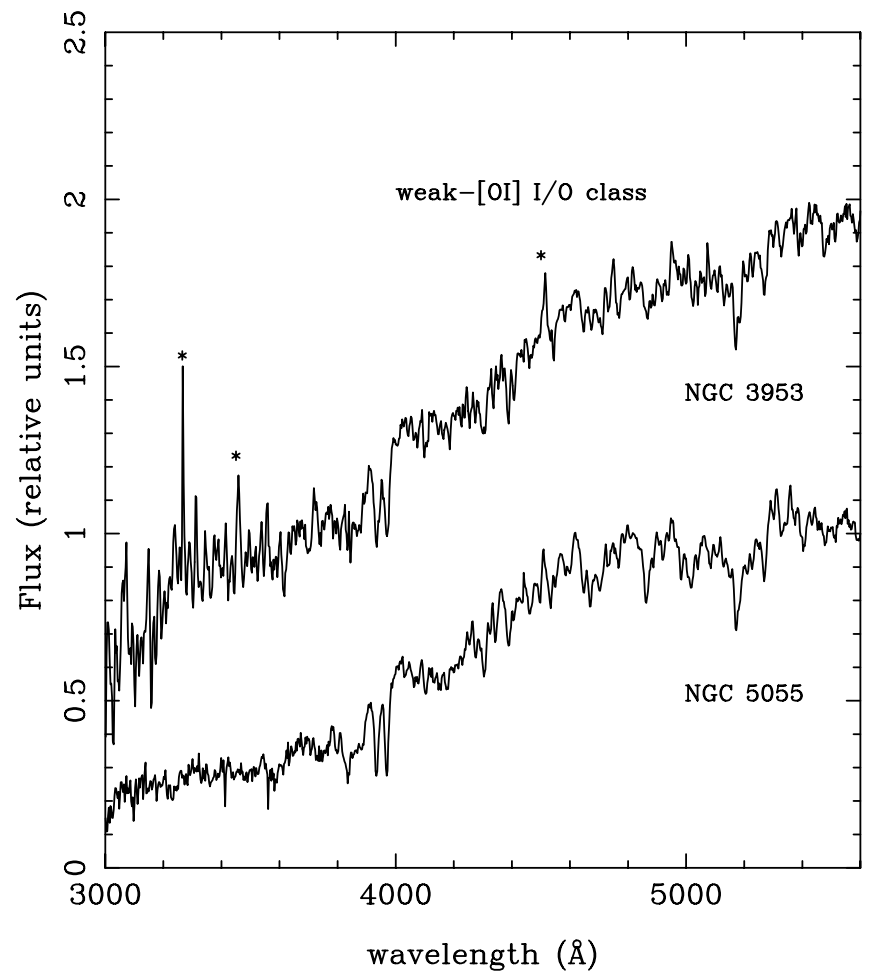

FIG. 5.-Nuclear spectra of weak-[O I] sources in the $I / O$ class, i.e., with a mixture of intermediate-age and older stars. Hot pixels in the spectra are labeled by an asterisk. 

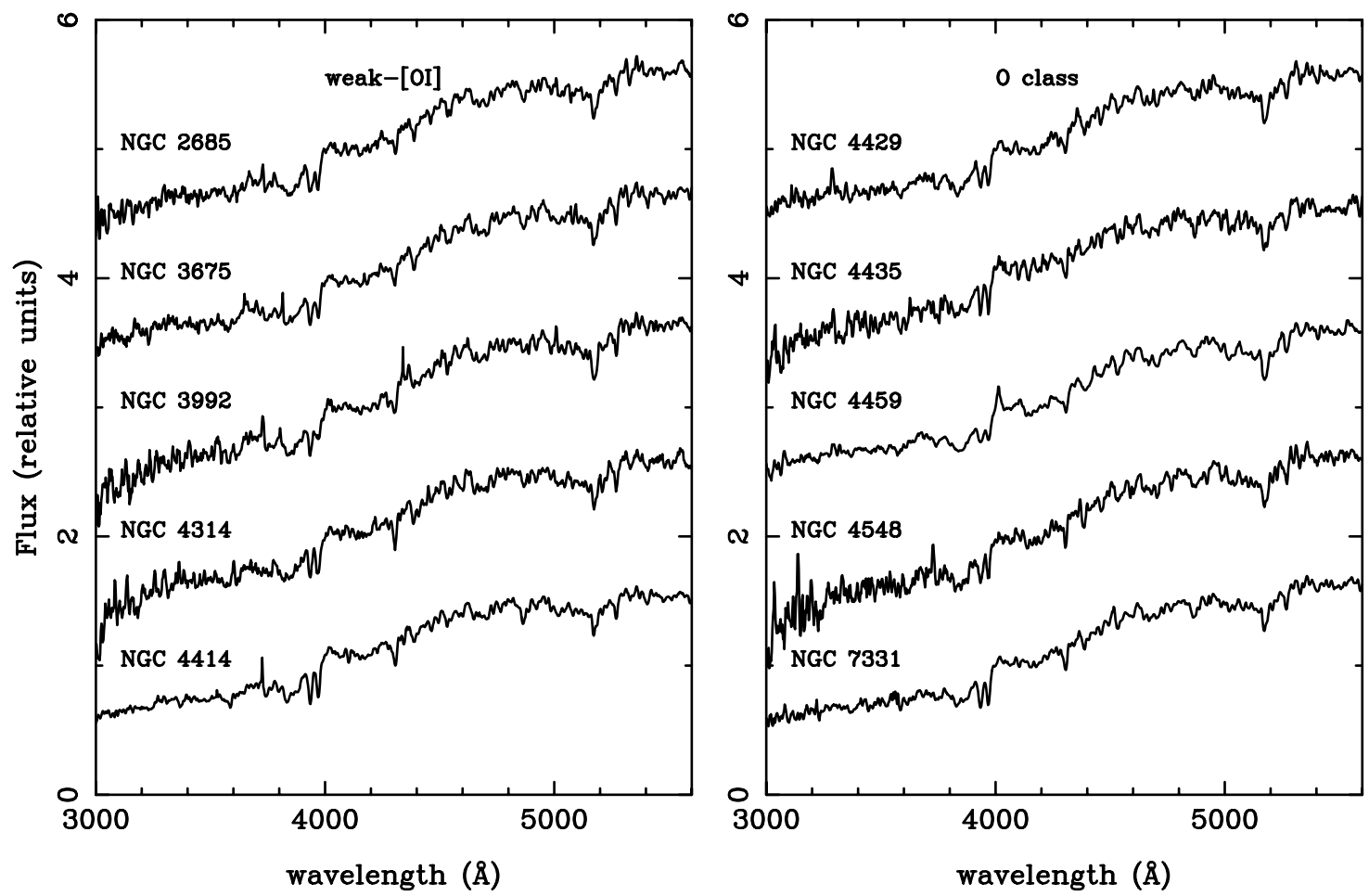

FIG. 6.-Nuclear spectra of weak-[O I] sources in the $O$ class, i.e., with a predominantly old population

detected. This result is also maintained when the spectra are subtracted from the intermediate-age and old stellar components (obtained through the template decomposition discussed above). Only in NGC 3507 do we find marginal evidence of a broad feature at $4620 \AA$ in the residual spectrum, although no broad feature at $4680 \AA$ is detected.

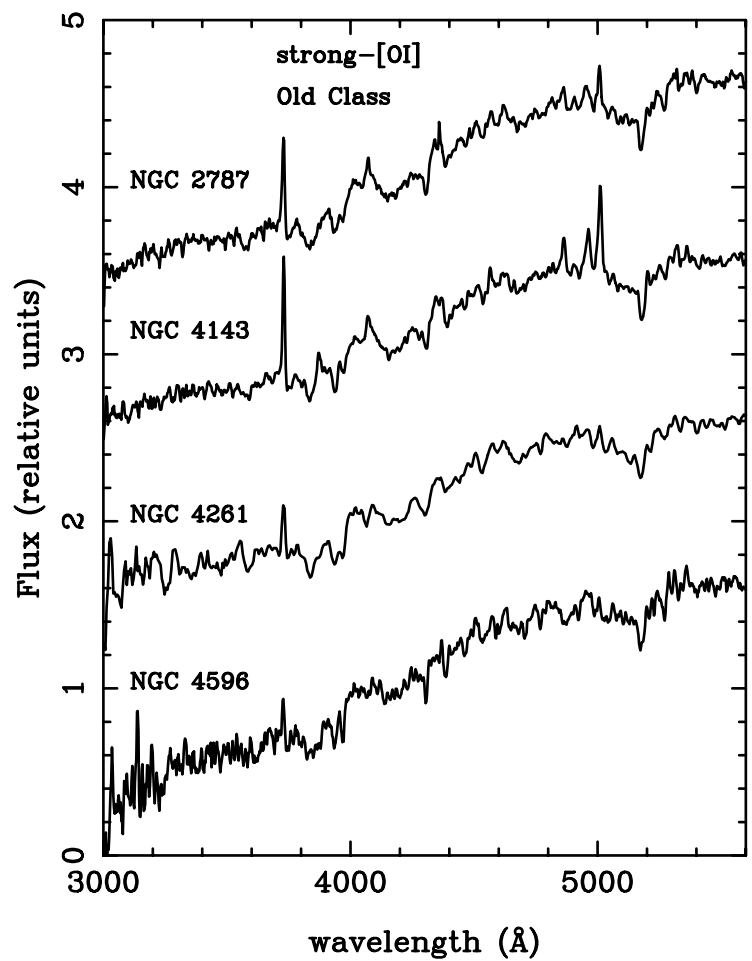

Most of the LLAGN spectra are dominated by starlight, with weak or absent nebular emission. Three LINERs (NGC 3998, NGC 4203, and NGC 4450), which are classified as L1.9 for presenting broad Balmer lines, are the exception (Fig. 7, right). These objects are among the strongest $\left[\mathrm{O}_{\mathrm{I}}\right] / \mathrm{H} \alpha$ emitters of the HFS97 sample, with values of $0.53,1.22$, and 0.67 ,

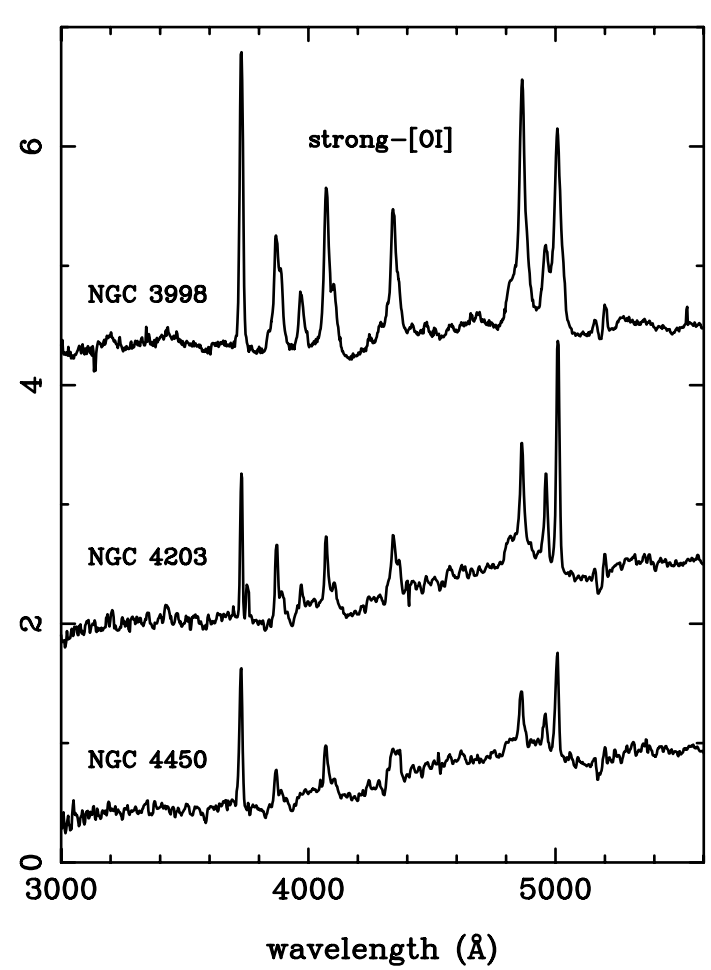

FIG. 7.-Nuclear spectra of strong-[O I ] sources: in the $O$ class (left), i.e., with a predominantly old population, and with diluted stellar lines (right). 
respectively. Another important feature of these objects is that the stellar lines are very diluted with respect to the strength of these lines in a typical old and/or intermediateage population. This resembles what is observed in many Seyfert 2 galaxies (Schmitt et al. 1999; González Delgado, Heckman, \& Leitherer 2001; Cid Fernandes et al. 2001a) in which the optical continuum is modeled by an old population plus a featureless continuum (fitted by a power law). In $\S 5$ we discuss the origin of the continuum in these LINERs. None of the weak-[O I] LLAGNs observed have such properties.

We find that an intermediate-age population is more frequent in TOs (7/17) than in LINERs $(2 / 11)$. Note, however, that both NGC 3368 and NGC 3507, the two $\eta=I$ LINERs, have $\left[\mathrm{O}_{\mathrm{I}}\right] / \mathrm{H} \alpha=0.18$, therefore trespassing the LINER/TO boundary of HFS97 by an insignificant amount. Expressed in terms of our weak- and strong-[ $\left[\begin{array}{ll}\mathrm{O}_{1}\end{array}\right]$ classes (divided at $[\mathrm{O} \mathrm{I}] / \mathrm{H} \alpha=0.25)$, this difference becomes even more pronounced: 9/21 weak-[O I] nuclei but none of the strong-[O I] nuclei in the STIS sample present HOBLs. Taking these numbers together with the data from Paper I, we find that only 2 (NGC 841, [O I] $/ \mathrm{H} \alpha=0.58$, and NGC 5005, $[\mathrm{O}$ I $] / \mathrm{H} \alpha=0.65)$ out of the $24(8 \%)$ strong-[O I] LLAGNs belong to the $\eta=I$ class, while this fraction is $20 / 47(42 \%)$ for weak-[O I] LLAGNs. As pointed out in Paper I, these numbers suggest a possible link between the central stellar population and emission-line properties.

Figure 8 shows the STIS spectra of four $\mathrm{H}$ II nuclei (NGC 278, NGC 3351, NGC 4245, and NGC 4800) and one nonactive galaxy (NGC 1023) to be compared with the LLAGNs. Paradoxically, not all the H II nuclei show a continuum dominated by young and/or intermediate-age populations! In fact, the dominant central $0.3 \times 00^{\prime \prime} 2$ stellar population in NGC 4245 and NGC 4800 is old $\left(\gtrsim 10^{10} \mathrm{yr}\right)$ and somewhat similar to the nonactive galaxy NGC 1023. However, the $\mathrm{H} \alpha$ distribution in both objects is extended and more prominent in the off-nuclear spectra than in the central

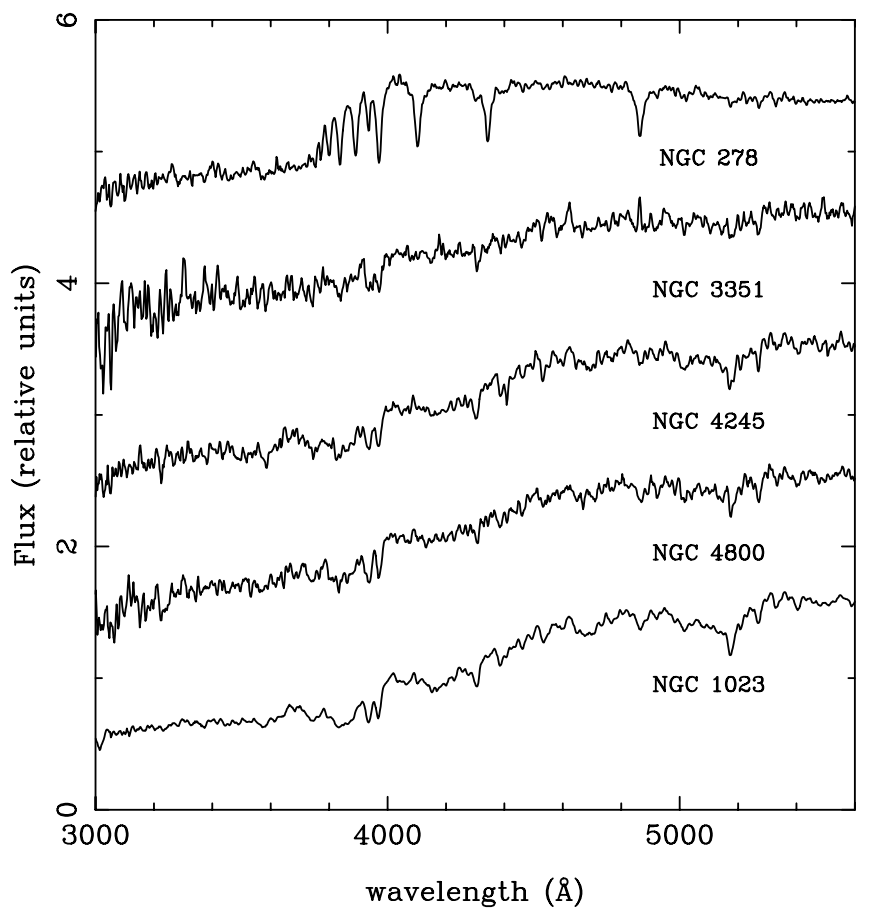

FIg. 8.-Nuclear spectra of a nonactive galaxy, NGC 1023, and four H II nuclei.
$0.3 \times 0 . " 2$, where $\mathrm{H} \alpha$ is in absorption. It would be interesting to revise the emission-line classification of these objects and to check if their $\mathrm{H}$ II denomination is associated with the large aperture of the spectra used in the HFS97 classification; then it is more associated with star formation located in the circumnuclear region $(100 \mathrm{pc}$ to $1 \mathrm{kpc})$ than in the nucleus. This comparison also indicates the relevance of the central morphology of the continuum and emission lines in the classification of LLAGNs.

\subsection{Spectral Properties}

In this section we present measurements of spectral indices indicative of stellar populations for the STIS spectra. We list the measurements only for extractions $a$, since in most of the objects the differences in the spectral indices with respect to extractions $b$ are small. We have measured seven equivalent widths and two continuum colors in the system of Bica \& Alloin (1986a, 1986b): $W_{\mathrm{C}}, W_{\mathrm{wlb}}, W_{\mathrm{K}}, W_{\mathrm{H}}, W_{\mathrm{CN}}, W_{\mathrm{G}}, W_{\mathrm{Mg}}$, $F_{3660} / F_{4020}$, and $F_{4510} / F_{4510}$. These measurements were performed following the automated procedure devised in Paper I except for three cases (NGC 4459, NGC 4569, and NGC 6503), where the pseudocontinuum was corrected by hand. We have also measured the $4000 \AA$ freak index $\left[D_{n}(4000)\right]$ and the [O II] and $\mathrm{H} \delta\left(\mathrm{H} \delta_{A}\right)$ equivalent widths following recipes in Balogh et al. (1999) and Worthey \& Ottaviani (1997).

Table 2 lists the results of these measurements. In Figure 9 we examine the relation between $D_{n}(4000), \mathrm{H} \delta_{A}$, and $W_{\mathrm{K}}$ for the combined STIS plus ground-based samples. Different symbols correspond to stellar population classes $\eta=I, I / O$, and $O$ (circles, triangles, and squares, respectively). Filled and open symbols are used for STIS and ground-based observations, respectively. As discussed in Paper I, there is an excellent agreement between the empirical stellar population classes and the spectral indices. Nuclei with young and/or intermediate-age populations $(\eta=I)$ have low $W_{\mathrm{K}}$ and $D_{n}(4000)$, while for nuclei dominated by an old stellar population these indices assume large values, with $\eta=I / O$ objects in between.

\section{EMPIRICAL POPULATION SYNTHESIS ANALYSIS}

\subsection{The Method}

In order to quantify the stellar population mixture of LLAGNs, we use the EPS algorithm described in Cid Fernandes et al. (2001b). Briefly, the code synthesizes a set of equivalent widths and colors by means of a combination of a base of 12 observed star clusters of different ages and metallicities (Schmidt et al. 1991; Bica \& Alloin 1986a, 1986b) plus an $F_{\nu} \propto \nu^{-1.5}$ power law to represent an AGN featureless continuum (FC). The output of the code consists of a population vector $\boldsymbol{x}$, whose components represent flux fractions associated with each population in the base, plus the $V$-band extinction, modeled as due to a uniform dust screen. These parameters correspond to a likelihood-weighted mean of $10^{8}$ combinations obtained from a Metropolis tour through the $\left(\boldsymbol{x}, A_{V}\right)$-space. We use as input to the EPS code five spectral indices: $W_{\mathrm{K}}, W_{\mathrm{CN}}$, and $W_{\mathrm{G}}$ plus the $F_{3660} / F_{4020}$ and $F_{4510} / F_{4020}$ colors. This same set of observables was used in the EPS analysis of starburst (Cid Fernandes, Leão, \& Rodrigues-Lacerda 2003) and Seyfert 2 galaxies (Cid Fernandes et al. 2001a), which provide an important reference for comparison.

The virtues and shortcomings of EPS have been extensively discussed in Cid Fernandes et al. (2001b, 2003). Our experience 
TABLE 2

Spectral Properties in the $a$ LLAGN STIS/CCD (G430L) Spectra

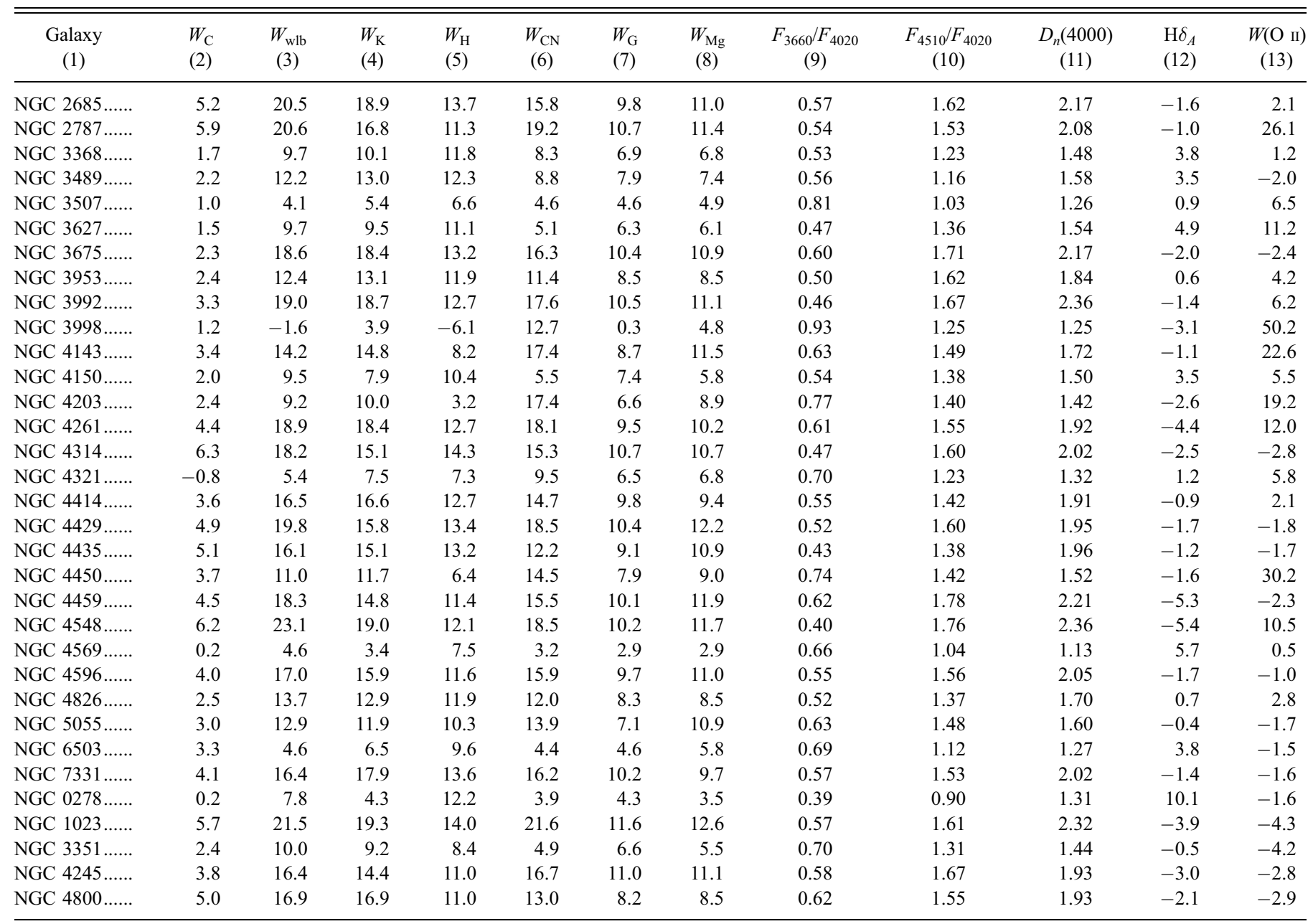

Notes.-Col. (1): Galaxy name. Cols. (2)-(10): Equivalent widths of seven absorption features and two colors, all in the Bica \& Alloin system. Col. (11): $4000 \AA$ break index (Balogh et al. 1999). Col. (12): $\mathrm{H} \delta_{A}$ equivalent width of Worthey \& Ottaviani 1997. Col. (13): [O II] equivalent width of Balogh et al. 1999.

with this method in a variety of studies has taught us that it is a superb tool to analyze stellar population mixtures provided that one keeps the description at a relatively coarse level. Accordingly, in this paper we group components of the same age in a reduced population vector $\boldsymbol{x}=\left(x_{\mathrm{FC}}, x_{6}, x_{7}, x_{8}, x_{9}, x_{10}\right)$, where $x_{6}-x_{10}$ correspond to five logarithmically spaced ages of $10^{6}$, $10^{7}, 10^{8}, 10^{9}$, and $10^{10} \mathrm{yr}$, and $x_{\mathrm{FC}}$ corresponds to the powerlaw component. Even this description is too finely graded given the limitations of the EPS imposed by the combination of observational errors, limited input information, and quasilinear dependencies within the base. We therefore base our analysis of the EPS results on an even coarser (but more robust) description obtained by further grouping similar $\boldsymbol{x}$ components. For instance, although the method does not distinguish well between the $10^{6}, 10^{7}$, and FC individual components, their sum $x_{Y / \mathrm{FC}} \equiv x_{\mathrm{FC}}+x_{6}+x_{7}$ is well constrained. Similar rebinnings of the remaining components are employed in the discussion below.

We have also calculated formal errors and covariances for three condensed EPS fractions $\left(x_{Y / \mathrm{FC}}, x_{8}\right.$, and $\left.x_{9+10}\right)$. The average errors are $2.2 \%, 3.2 \%$, and $3.2 \%$, respectively. These errors are low and allow us to distinguish between the different components with a precision of about 3\%. However, this does not apply for old systems with a contribution of $x_{9+10} \geq 90 \%$. In these objects, the fractions $x_{Y / F C}$ and $x_{8}$ obtained are similar to the formal errors, and consequently these values are not significant.

\subsection{Statistical Results}

Tables 3 and 4 list the EPS results for both the ground-based and STIS samples, respectively. The population vector is expressed as the percentage fraction of the flux at a normalization wavelength of $4020 \AA$. In this section we present histograms of the results of the synthesis grouped in three age bins, used to represent old population $\left(10^{10} \mathrm{yr}, x_{10}\right)$, intermediate-age population $\left(10^{8}-10^{9} \mathrm{yr}, x_{8}+x_{9}\right)$, and young plus FC components $\left(x_{Y / F C}\right)$. In these histograms we divide the LLAGNs into weak-[O I] $([\mathrm{O} \mathrm{I}] / \mathrm{H} \alpha \leq 0.25)$ and strong-[O I] $([\mathrm{O} \mathrm{I}] / \mathrm{H} \alpha \geq 0.25)$ sources.

Figure 10 shows the results for the ground-based observations only. The average (median) values of the distributions of the old, intermediate-age, and young stellar populations of the weak-[O I] LLAGNs are 48\% (47\%), 46\% (45\%), and 6\% (5\%), respectively. For the strong-[O I] LLAGNs these values are $62 \%(64 \%), 35 \%(33 \%)$, and $3 \%(3 \%)$. A remarkable result from this analysis is that the $Y / \mathrm{FC}$ component rarely exceeds $10 \%$ of the flux in the $1.1 \times 1^{\prime \prime}$ ground-based aperture. This only happens in 5/51 (10\%) of our LLAGNs 


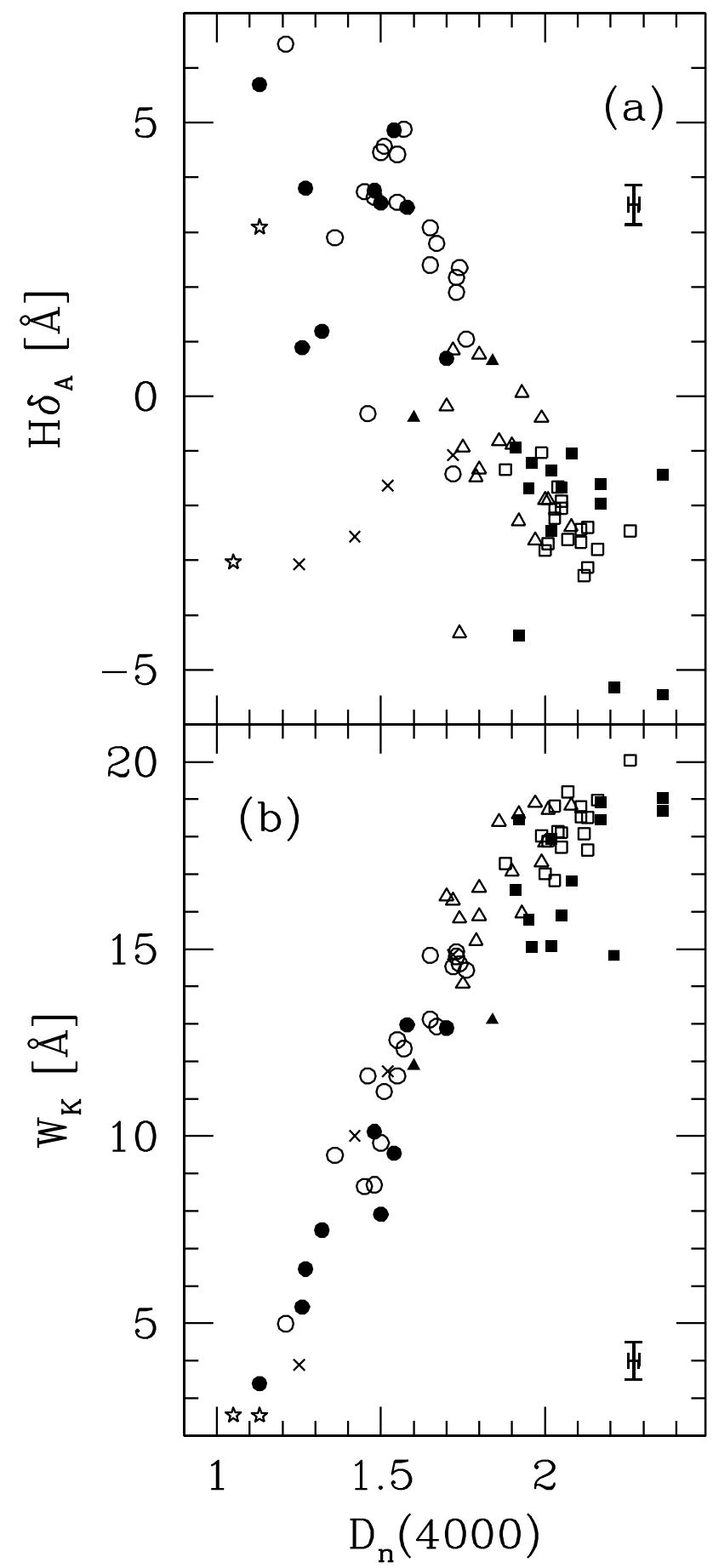

FIG. 9.- Relations between spectral indices: $D_{n}(4000), \mathrm{H} \delta_{A}$, and $W_{\mathrm{K}}$. The stellar population classes $I, I / O$, and $O$ are represented by circles, triangles, and squares, respectively. Except for the two stars, which represent the H II nuclei NGC 3367 and NGC 6217 from Paper I, only LLAGNs are included. Crosses are used to represent NGC 3998, NGC 4143, NGC 4203, and NGC 4450, the four STIS sources with strong emission lines (Fig. 7, right). Filled and open symbols are used for STIS and ground-based observations, respectively. [See the electronic edition of the Journal for a color version of this figure.]

(NGC 772, NGC 4569, NGC 5377, NGC 5678, and NGC 6503), all of which are weak-[O I] sources. Another significant difference between strong and weak $\left[\begin{array}{ll}\mathrm{O}_{\mathrm{I}} & \mathrm{I}\end{array}\right.$ is found in the fraction of intermediate-age population. The fraction of weak[O I] LLAGNs with $x_{I}=x_{8}+x_{9}>35 \%$ is $25 / 34(74 \%)$, but only 5/17 (29\%) for strong [O I]. Regarding the old stellar population, the fraction of weak-[O I] nuclei with $x_{10}<65 \%$ is $27 / 34(79 \%)$, and $10 / 17(59 \%)$ for strong [ [ I I]. These numbers essentially translate the analysis performed in Paper I to population strengths, and they clearly indicate that weak[O I] LLAGNs are nuclei with an important intermediate-age population.

Figure 11 shows the results for the STIS $a$ spectra. The EPS analysis has also been performed for the $b$ spectra, but we have not found a significant difference between the results for the two apertures. The previous conclusion that weak-[O I] LLAGNs have a larger contribution from the intermediate-age population than strong-[O I] LLAGNs is still valid. In 16 of the $21(76 \%)$ weak-[O I] LLAGNs but only $2 / 7$ (28\%) of the strong-[O I] LLAGNs is the contribution of the intermediateage population larger than $35 \%$. However, there is a noticeable difference with respect to the ground-based observations, related to the strength of the young plus FC component. The mean value of this component is $x_{Y / \mathrm{FC}}=12 \%$ and $22 \%$ for weak - and strong-[O I] LLAGNs, respectively. These values are larger than the mean contributions in the ground-based spectra, $3 \%$ and $6 \%$, respectively. There are four weak-[O I] (NGC 3507, NGC 4321, NGC 4569, and NGC 6503) and three strong-[O I] LLAGNs (NGC 3998, NGC 4203, and NGC 4450) with $x_{Y / \mathrm{FC}}>20 \%$, whereas only NGC 4569 has such a strong component in the ground-based data. These three LINERs show very strong emission lines and diluted stellar lines, probably produced by the contribution from an AGN component (see $\S 5.1$ ). On the other hand, in NGC 3507, NGC 4321, NGC 4569, and NGC 6503, the UV emission and the spectral characteristics of the optical spectra suggest that the large contribution from the $Y / \mathrm{FC}$ component is provided by a young stellar cluster. This cluster must be compact, as indicated by the morphology in the WFPC2 images and surface brightness profile along the slit, and hence its contribution to the optical light is much less important in a ground-based aperture. It is interesting to note that in the ground-based aperture, NGC 4569 and NGC 6503 are outstanding for their large intermediate-age population.

\section{DISCUSSION}

\subsection{Comparison with Nonactive Galaxies}

The EPS analysis suggests that many of the LLAGNs have a $10^{8}-10^{9} \mathrm{yr}$ intermediate-age stellar population that is far more conspicuous in weak-[O $\mathrm{O}]$ than in strong-[O $\mathrm{I}]$ nuclei. The question arises whether these statistics are the typical stellar population of early-type galaxies or whether intermediateage stars are more significant in LLAGNs than in nonactive galaxies of the same morphological type.

Raimann et al. (2003) have analyzed the stellar population of a small sample of S0-Sbc nonactive galaxies using the same EPS code and star cluster base. They find that the old and the intermediate-age population contributes with $x_{O}=65 \%$ and $x_{I}=35 \%$ to the continuum at $4020 \AA$. The mean contributions in our ground-based aperture are $x_{O}=62 \%$ and $x_{I}=35 \%$ for strong-[O $\left.\mathrm{I}\right]$ LLAGNs and $x_{O}=48 \%$ and $x_{I}=46 \%$ for weak-[O I] LLAGNs. On the other hand, the intermediate-age population contributes with $x_{I} \geq 35 \%$ in $74 \%$ of the weak-[O I] LLAGNs, while this happens in only $29 \%$ of the strong-[O I] LLAGNs. These results suggest that weak-[O I] LLAGNs have an intermediate-age population that is more significant than in nonactive galaxies and have 
TABLE 3

EPS Results for the Ground-Based Observations

\begin{tabular}{|c|c|c|c|c|c|c|c|}
\hline Galaxy & $x_{10}$ & $x_{9}$ & $x_{8}$ & $x_{7}$ & $x_{6}$ & $x_{\mathrm{PL}}$ & $A_{V}$ \\
\hline NGC $0266 \ldots \ldots$. & 63.3 & 33.0 & 1.8 & 1.0 & 0.4 & 0.4 & 0.28 \\
\hline NGC $0315 \ldots \ldots$. & 68.2 & 23.3 & 3.6 & 2.6 & 1.2 & 1.1 & 0.34 \\
\hline NGC $0404 \ldots \ldots$. & 27.3 & 35.7 & 28.7 & 4.4 & 1.9 & 1.9 & 1.56 \\
\hline NGC $0410 \ldots \ldots$ & 65.6 & 27.7 & 3.1 & 1.9 & 0.8 & 0.8 & 0.30 \\
\hline NGC $0428 \ldots \ldots$ & 25.6 & 64.4 & 6.3 & 2.1 & 0.8 & 0.9 & 0.79 \\
\hline NGC $0521 \ldots \ldots$. & 61.4 & 33.4 & 2.7 & 1.3 & 0.6 & 0.6 & 0.56 \\
\hline NGC $0660 \ldots \ldots$ & 42.0 & 48.4 & 5.1 & 2.5 & 1.0 & 1.1 & 2.37 \\
\hline NGC $0718 \ldots \ldots$ & 36.3 & 46.1 & 11.6 & 3.3 & 1.4 & 1.4 & 0.74 \\
\hline NGC $0772 \ldots \ldots$ & 46.9 & 24.4 & 11.5 & 8.7 & 4.9 & 3.5 & 0.28 \\
\hline NGC $0841 \ldots \ldots$ & 39.5 & 49.9 & 6.1 & 2.5 & 1.0 & 1.1 & 0.57 \\
\hline NGC $1052 \ldots \ldots$ & 70.1 & 20.8 & 3.9 & 2.8 & 1.2 & 1.1 & 0.37 \\
\hline NGC $1161 \ldots \ldots$. & 75.5 & 20.9 & 1.8 & 1.0 & 0.4 & 0.4 & 0.24 \\
\hline NGC $1169 \ldots \ldots$ & 73.2 & 22.9 & 1.7 & 1.2 & 0.5 & 0.5 & 0.15 \\
\hline NGC $2681 \ldots \ldots$. & 25.7 & 52.7 & 16.1 & 3.0 & 1.2 & 1.3 & 0.91 \\
\hline NGC $2685 \ldots \ldots$. & 65.2 & 31.3 & 1.8 & 1.0 & 0.4 & 0.4 & 0.26 \\
\hline NGC $2911 \ldots \ldots$. & 64.4 & 29.7 & 3.1 & 1.5 & 0.7 & 0.6 & 0.80 \\
\hline NGC $3166 \ldots \ldots$. & 42.9 & 48.3 & 4.9 & 2.1 & 0.9 & 0.9 & 0.62 \\
\hline NGC $3169 \ldots \ldots$ & 50.2 & 44.2 & 3.0 & 1.5 & 0.6 & 0.6 & 1.15 \\
\hline NGC $3226 \ldots . .$. & 78.3 & 16.4 & 2.4 & 1.5 & 0.7 & 0.6 & 0.53 \\
\hline NGC $3245 \ldots \ldots$. & 59.4 & 27.4 & 5.2 & 4.2 & 2.0 & 1.8 & 0.33 \\
\hline NGC $3627 \ldots \ldots$. & 29.6 & 47.1 & 16.7 & 3.6 & 1.4 & 1.5 & 1.14 \\
\hline NGC $3705 \ldots \ldots$ & 36.7 & 52.6 & 6.5 & 2.3 & 0.9 & 1.0 & 1.08 \\
\hline NGC $4150 \ldots \ldots$ & 29.2 & 52.3 & 12.7 & 3.2 & 1.2 & 1.3 & 0.62 \\
\hline NGC $4192 \ldots \ldots$. & 52.6 & 38.1 & 4.5 & 2.6 & 1.1 & 1.1 & 1.77 \\
\hline NGC $4438 \ldots \ldots$ & 67.2 & 27.2 & 2.8 & 1.5 & 0.7 & 0.6 & 1.15 \\
\hline NGC $4569 . . . .$. & 19.6 & 13.2 & 45.7 & 11.0 & 5.0 & 5.4 & 0.35 \\
\hline NGC $4736 \ldots \ldots$. & 36.8 & 43.9 & 12.6 & 3.7 & 1.6 & 1.5 & 0.68 \\
\hline NGC $4826 \ldots \ldots$ & 44.6 & 40.1 & 9.6 & 3.1 & 1.3 & 1.3 & 0.67 \\
\hline NGC $5005 \ldots \ldots$. & 34.4 & 53.4 & 7.4 & 2.6 & 1.0 & 1.1 & 1.38 \\
\hline NGC $5055 \ldots \ldots$ & 46.1 & 34.1 & 12.4 & 4.0 & 1.7 & 1.7 & 0.81 \\
\hline NGC $5377 \ldots \ldots$. & 31.1 & 24.3 & 32.5 & 6.2 & 3.1 & 2.8 & 0.64 \\
\hline NGC $5678 \ldots \ldots$. & 34.8 & 31.8 & 22.1 & 5.9 & 2.7 & 2.8 & 1.85 \\
\hline NGC $5879 \ldots \ldots$ & 33.7 & 60.5 & 3.3 & 1.4 & 0.5 & 0.6 & 0.89 \\
\hline NGC $5921 \ldots \ldots$. & 29.8 & 45.2 & 17.6 & 4.1 & 1.6 & 1.8 & 0.68 \\
\hline NGC $5970 \ldots \ldots$. & 60.7 & 35.8 & 1.6 & 1.0 & 0.4 & 0.5 & 0.18 \\
\hline NGC $5982 \ldots \ldots$. & 58.7 & 36.7 & 2.2 & 1.3 & 0.5 & 0.5 & 0.21 \\
\hline NGC $5985 \ldots \ldots$. & 65.0 & 31.4 & 1.7 & 1.0 & 0.4 & 0.5 & 0.22 \\
\hline NGC $6340 \ldots \ldots$ & 85.4 & 12.0 & 1.3 & 0.7 & 0.3 & 0.3 & 0.91 \\
\hline NGC $6384 \ldots \ldots$. & 69.1 & 27.4 & 1.4 & 1.1 & 0.5 & 0.5 & 0.13 \\
\hline NGC $6482 \ldots \ldots$. & 69.7 & 26.4 & 1.7 & 1.2 & 0.5 & 0.5 & 0.13 \\
\hline NGC $6500 \ldots \ldots$ & 60.8 & 26.4 & 3.6 & 4.9 & 2.4 & 1.8 & 0.12 \\
\hline NGC $6501 \ldots . .$. & 63.1 & 28.3 & 4.4 & 2.2 & 1.0 & 0.9 & 0.42 \\
\hline NGC $6503 \ldots . .$. & 39.3 & 31.4 & 12.6 & 8.7 & 3.9 & 4.1 & 0.43 \\
\hline NGC $6702 \ldots \ldots$ & 62.8 & 32.4 & 2.1 & 1.4 & 0.6 & 0.6 & 0.21 \\
\hline NGC $6703 \ldots . .$. & 71.3 & 24.3 & 2.1 & 1.2 & 0.5 & 0.5 & 0.31 \\
\hline NGC $6951 \ldots . .$. & 51.2 & 40.8 & 4.5 & 1.9 & 0.8 & 0.8 & 0.37 \\
\hline NGC $7177 \ldots \ldots$ & 60.5 & 32.4 & 3.4 & 2.0 & 0.9 & 0.9 & 0.57 \\
\hline NGC $7217 \ldots \ldots$ & 75.7 & 20.8 & 1.7 & 0.9 & 0.4 & 0.4 & 0.41 \\
\hline NGC $7331 \ldots \ldots$ & 61.8 & 33.3 & 2.5 & 1.3 & 0.6 & 0.6 & 0.43 \\
\hline NGC $7626 \ldots \ldots$ & 69.4 & 25.1 & 2.6 & 1.6 & 0.7 & 0.6 & 0.27 \\
\hline NGC $7742 \ldots \ldots$ & 52.3 & 41.6 & 3.2 & 1.5 & 0.6 & 0.7 & 0.64 \\
\hline NGC $3367 \ldots \ldots$. & 9.1 & 6.4 & 5.3 & 31.9 & 32.5 & 14.8 & 0.11 \\
\hline NGC $6217 \ldots \ldots$ & 11.2 & 6.7 & 34.2 & 16.5 & 17.4 & 13.9 & 0.26 \\
\hline NGC $0205 \ldots \ldots$. & 25.4 & 24.5 & 38.5 & 5.9 & 2.8 & 2.8 & 0.60 \\
\hline NGC $0221 \ldots \ldots$. & 56.4 & 39.1 & 2.1 & 1.3 & 0.6 & 0.6 & 0.20 \\
\hline NGC $0224 \ldots \ldots$ & 67.1 & 25.5 & 3.2 & 2.3 & 1.0 & 0.9 & 0.22 \\
\hline NGC $0628 \ldots \ldots$. & 55.8 & 37.4 & 2.9 & 2.0 & 0.8 & 0.9 & 0.35 \\
\hline NGC $1023 \ldots \ldots$ & 79.6 & 17.3 & 1.5 & 0.9 & 0.4 & 0.4 & 0.16 \\
\hline NGC $2950 \ldots \ldots$. & 60.0 & 33.4 & 3.1 & 1.9 & 0.8 & 0.8 & 0.23 \\
\hline NGC $6654 \ldots \ldots$ & 75.2 & 20.6 & 1.9 & 1.3 & 0.6 & 0.5 & 0.14 \\
\hline
\end{tabular}


TABLE 4

EPS Results For the STIS $a$ Data

\begin{tabular}{|c|c|c|c|c|c|c|c|}
\hline Galaxy & $x_{10}$ & $x_{9}$ & $x_{8}$ & $x_{7}$ & $x_{6}$ & $x_{\mathrm{PL}}$ & $A_{V}$ \\
\hline NGC $2685 \ldots \ldots$ & 66.4 & 29.4 & 2.0 & 1.2 & 0.5 & 0.5 & 0.51 \\
\hline NGC $2787 \ldots \ldots$. & 60.5 & 31.4 & 4.4 & 2.1 & 0.9 & 0.8 & 0.38 \\
\hline NGC $3368 \ldots \ldots$ & 33.1 & 31.7 & 24.8 & 5.5 & 2.5 & 2.4 & 0.73 \\
\hline NGC $3489 \ldots \ldots$ & 32.1 & 50.5 & 10.9 & 3.5 & 1.4 & 1.5 & 0.31 \\
\hline NGC $3507 \ldots \ldots$ & 26.8 & 14.4 & 14.7 & 17.4 & 15.2 & 11.5 & 0.25 \\
\hline NGC $3627 \ldots \ldots$. & 31.7 & 36.5 & 22.3 & 5.1 & 2.2 & 2.2 & 1.66 \\
\hline NGC $3675 \ldots \ldots$ & 74.1 & 20.3 & 2.5 & 1.7 & 0.7 & 0.7 & 0.59 \\
\hline NGC $3953 \ldots \ldots$. & 42.5 & 36.5 & 12.8 & 4.4 & 1.9 & 1.9 & 1.72 \\
\hline NGC $3992 \ldots \ldots$. & 59.1 & 37.4 & 1.9 & 0.9 & 0.4 & 0.4 & 0.91 \\
\hline NGC $3998 \ldots \ldots$. & 15.5 & 5.8 & 5.7 & 33.7 & 20.7 & 18.6 & 1.47 \\
\hline NGC $4143 \ldots \ldots$ & 57.0 & 24.7 & 8.5 & 5.3 & 2.4 & 2.1 & 0.43 \\
\hline NGC $4150 \ldots \ldots$ & 39.3 & 20.6 & 22.0 & 7.9 & 5.7 & 4.5 & 1.48 \\
\hline NGC $4203 \ldots \ldots$. & 48.9 & 8.6 & 9.0 & 15.3 & 11.9 & 6.3 & 0.51 \\
\hline NGC $4261 \ldots \ldots$ & 65.4 & 29.2 & 2.5 & 1.6 & 0.6 & 0.7 & 0.25 \\
\hline NGC $4314 \ldots \ldots$ & 51.7 & 34.8 & 8.6 & 2.6 & 1.2 & 1.1 & 1.10 \\
\hline NGC $4321 \ldots \ldots$. & 35.5 & 12.9 & 19.0 & 13.3 & 11.5 & 7.7 & 0.56 \\
\hline NGC $4414 \ldots \ldots$ & 48.0 & 44.0 & 4.2 & 2.0 & 0.8 & 0.8 & 0.44 \\
\hline NGC $4429 \ldots \ldots$ & 59.4 & 28.0 & 7.6 & 2.7 & 1.2 & 1.1 & 0.72 \\
\hline NGC $4435 \ldots \ldots$ & 27.8 & 61.6 & 7.1 & 1.9 & 0.8 & 0.8 & 1.17 \\
\hline NGC $4450 \ldots \ldots$ & 53.4 & 13.6 & 8.4 & 12.1 & 7.6 & 5.0 & 0.41 \\
\hline NGC $4459 \ldots \ldots$ & 65.4 & 17.2 & 6.3 & 5.6 & 3.1 & 2.3 & 1.25 \\
\hline NGC $4548 \ldots \ldots$ & 57.9 & 39.1 & 1.6 & 0.7 & 0.3 & 0.3 & 1.26 \\
\hline NGC $4569 \ldots \ldots$ & 14.6 & 9.1 & 40.9 & 13.2 & 11.1 & 11.0 & 0.79 \\
\hline NGC $4596 \ldots \ldots$. & 54.0 & 34.3 & 6.4 & 2.9 & 1.2 & 1.2 & 0.79 \\
\hline NGC $4826 \ldots \ldots$ & 39.6 & 37.0 & 15.9 & 4.1 & 1.7 & 1.7 & 0.82 \\
\hline NGC $5055 \ldots \ldots$ & 44.0 & 24.0 & 14.1 & 9.5 & 4.5 & 3.9 & 1.08 \\
\hline NGC $6503 \ldots \ldots$ & 28.7 & 19.3 & 21.4 & 14.3 & 8.1 & 8.2 & 0.72 \\
\hline NGC $7331 \ldots \ldots$ & 60.4 & 34.1 & 2.7 & 1.5 & 0.6 & 0.6 & 0.39 \\
\hline NGC $0278 \ldots \ldots$ & 11.8 & 10.1 & 71.1 & 3.4 & 1.8 & 1.8 & 0.40 \\
\hline NGC $1023 \ldots \ldots$ & 78.7 & 18.3 & 1.3 & 0.9 & 0.4 & 0.3 & 0.12 \\
\hline NGC $3351 \ldots \ldots$ & 45.3 & 22.6 & 9.3 & 10.7 & 6.5 & 5.7 & 0.95 \\
\hline NGC $4245 \ldots \ldots$. & 64.8 & 16.3 & 9.0 & 5.0 & 2.9 & 2.0 & 0.89 \\
\hline NGC $4800 \ldots \ldots$ & 55.4 & 36.4 & 3.6 & 2.5 & 1.0 & 1.1 & 0.81 \\
\hline
\end{tabular}

less contribution of the old population than in nonactive galaxies.

\subsection{Emission Line versus Stellar Population}

In Paper I we found a strong tendency of the HOBLs to appear preferentially in weak-[O I] LLAGNs. As discussed in $\S 3.2$, this result also applies to the STIS observations. The detection of these lines implies the presence of a substantial intermediate-age population (e.g., González Delgado et al. 1999). This suggestion is confirmed by the EPS analysis performed here, which shows that LLAGNs with conspicuous HOBLs (i.e., those classified $\eta=I$ ) have $x_{8}+x_{9} \geq 30 \%$. The statistical connection between these populations and the weak-[O I] class is clearly illustrated in Figures 10 and 11 , which show that weak-[O I] LLAGNs have systematically higher contributions of intermediate-age populations. These
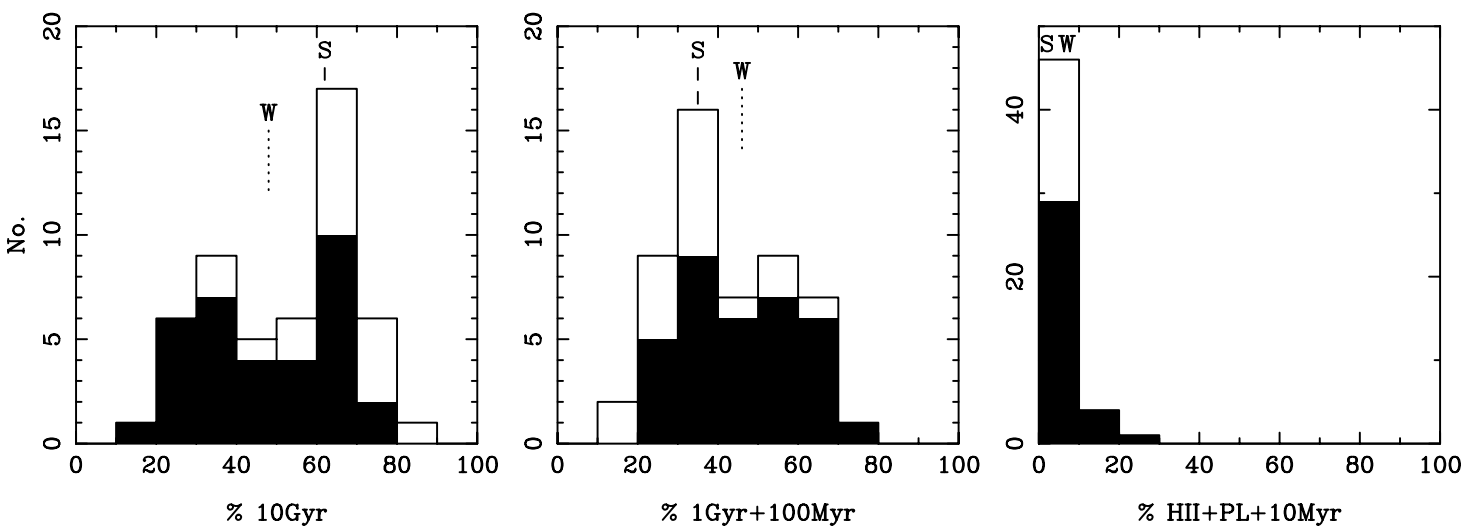

FIG. 10.- Histograms of the contribution of different age components, normalized to the light at $4020 \AA$. The histogram is for the ground-based observations, thus corresponding to stellar populations that contribute to a nuclear aperture of 1 "1 $1 \times 1$ ". The filled area represents the distributions of weak-[O I] LLAGNs. Labels " $\mathrm{W}$ " and " $\mathrm{S}$ " indicate the median corresponding to the distributions of weak- and strong-[O I] LLAGNs, respectively. 

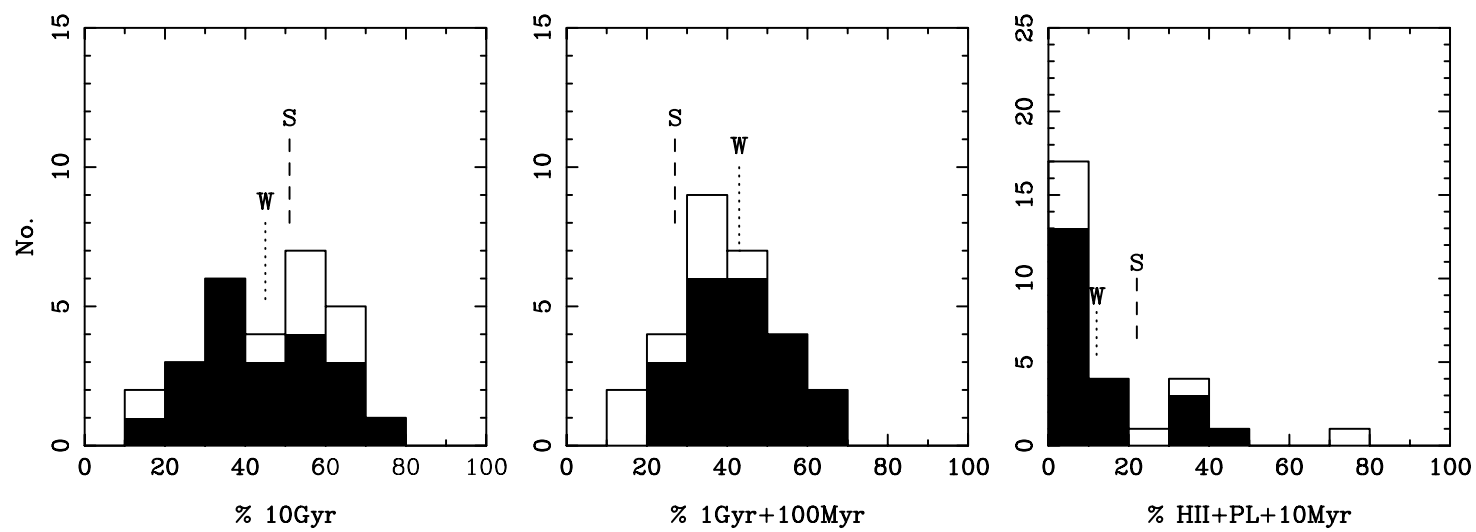

FIG. 11. - Same as Fig. 10, but for STIS $a$ spectra (corresponding to the nuclear $1^{\prime \prime} \times 0 . " 2$ )

results suggest a connection between LLAGN subtypes and their stellar population. Because these two classes differ in their emission-line properties, this connection implies a link between the stellar population and the ionization process in LLAGNs.

In Paper I we have noted a dichotomy between the [O I $] / \mathrm{H} \alpha$ ratio and the spectral indexes, which indicates that there are no strong-[O I] LLAGNs with conspicuous HOBLs and with $W_{\mathrm{K}} \leq 15 \AA$ and $W_{\mathrm{C}} \leq 3.5 \AA$. These results are also confirmed by the EPS analysis and are shown in Figure 12, where we plot $\left[\mathrm{O}_{\mathrm{I}}\right] / \mathrm{H} \alpha$ as a function of the fraction of the light provided by the old and the intermediate-age stellar populations. The $[\mathrm{O}$ I $] / \mathrm{H} \alpha$ versus $x_{O}$ diagram has an "inverted L" shape, which can be interpreted as the strong-[O I] LLAGNs having a stellar population mainly dominated by old stars, while weak$\left[\begin{array}{ll}\mathrm{O} & \mathrm{I}\end{array}\right]$ LLAGNs can have stellar populations of all ages. However, the $\left[\begin{array}{ll}\mathrm{O} & \mathrm{I}\end{array}\right] / \mathrm{H} \alpha$ versus $x_{I}$ diagram is L-shaped, showing that many of the weak-[O I] LLAGNs have higher $x_{I}$. This also confirms that most of the objects with intermediate age are weak in [O I]. As we suggested in Paper I, in these objects stellar sources can dominate the ionization, and we call them stellar LLAGNs.

We use the STIS spectra to look for these sources. The high spatial resolution provided by STIS is crucial to detect compact young stellar clusters, which otherwise can be masked by the underlying light emitted by the old stars in ground-based observations. The EPS analysis finds that only five weak-[O I] LLAGNs of the 34 observed from the ground have a young

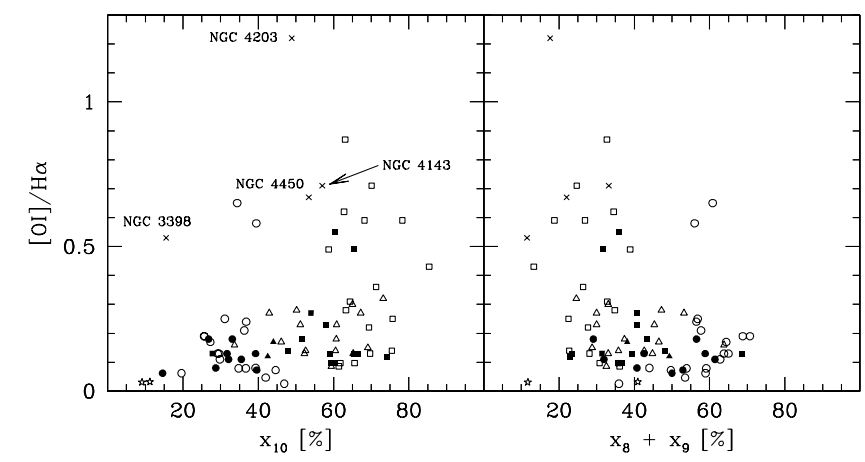

Fig. 12.- $[\mathrm{O}$ I $] / \mathrm{H} \alpha$ vs. fraction of the light provided by old $(10 \mathrm{Gyr}$; left) and intermediate-age $(1 \mathrm{Gyr}+100 \mathrm{Myr}$; right $)$ populations. Symbols are as in Fig. 9. [See the electronic edition of the Journal for a color version of this figure.] component with $x_{Y / \mathrm{FC}} \geq 10 \%$. These objects belong to the $I$ class and have $x_{I} \geq 30 \%$. However, the STIS EPS analysis finds a larger fraction of weak-[O I] LLAGNs with a young stellar component, in particular among those that belong to the $I$ class. In fact, seven of the nine weak-[O I] LLAGNs in the $I$ class have $x_{Y / \mathrm{FC}} \geq 10 \%$. In addition, two more weak-[O I] LLAGNs, NGC 5055 and NGC 4459, that belong to the $I / O$ and $O$ class, respectively, have $x_{Y / \mathrm{FC}} \geq 10 \%$. All of these objects have an important contribution of the intermediate-age stars. It is also important to note that in several objects in which the young stellar population was not detected in the ground-based observations, it has been detected in the STIS spectra. This suggests that some weak-[O I] LLAGNs that are classified as $I / O$ can have a very compact young cluster that is detected when observed with a small aperture, as is the case for NGC 5055.

Further evidence that these objects have young stellar clusters comes from the UV observations reported by Maoz et al. (1998). They found UV resonance lines formed in the wind of massive stars in NGC 404, NGC 4569, and NGC 5055, and possibly in NGC 6500. NGC 3507 also shows similar UV features (R. M. González Delgado et al. 2004, in preparation). All these are weak-[O I] LLAGNs and are outstanding at optical wavelengths for their intermediate-age population. Note, however, that only NGC 4569 has $x_{Y / \mathrm{FC}} \geq 10 \%$ in the groundbased aperture. We can thus conclude that weak-[ $\left[\begin{array}{ll}\mathrm{O} & \mathrm{I}\end{array}\right]$ LLAGNs that belong to the $I$ or $I / O$ class, and therefore have an important intermediate-age population, are stellar LLAGNs.

In the strong-[O I] LLAGNs, stars cannot play an important role in the gas ionization because we have not found a noticeable presence of stars younger than 1 Gyr. Photoionization by an AGN is likely the dominant emission-line mechanism in these sources. NGC 5005 could be the exception to this because it is a strong-[O I] LLAGN with HOBLs and so has an intermediate age. An AGN as the dominant source of ionization in LLAGNs can clearly be represented by the strong[O I] NGC 3998, NGC 4203, and NGC 4450, for which we have obtained $x_{O} \leq 50 \%$ and $x_{Y / \mathrm{FC}} \geq 20 \%$.

As pointed out by Cid Fernandes \& Terlevich (1995), a young stellar population has an optical continuum very similar in shape to a power law. There is thus a degeneracy between the young stellar and the power-law components in the EPS analysis. This degeneracy can be broken only at ultraviolet wavelengths. If the resonance lines of $\mathrm{C}$ IV $\lambda 1550$, Si IV $\lambda 1400$, and $N v \lambda 1240$ formed in the wind of massive stars are detected, then the optical light is provided by a young 
starburst. We have retrieved from the HST archive the UV spectrum of NGC 3998 to search for wind lines, but they are not present in the spectrum. On the contrary, broad C IV and $\mathrm{L} \alpha$ emission lines are detected. Furthermore, the optical spectrum shows also many $\mathrm{Fe}$ II multiplets, and the Balmer lines have a broad component. These spectral features suggest that a Seyfert 1 nucleus dwells in this strong-[O I] LLAGN. NGC 4203 and NGC 4450 have not been observed at the UV with the HST, so we cannot check for the presence of wind stellar lines. However, their optical spectra show doublepeaked broad H lines (Ho et al. 2000; Shields et al. 2000). This detection is interpreted as the presence of a nuclear accretion disk in these two galaxies. We thus conclude that the high $x_{Y / F C}$ component derived from the EPS analysis of these two galaxies is due to a nonthermal optical continuum that can be represented by a power law. Therefore, NGC 3998, NGC 4203, and NGC 4450 are strong-[O I] LLAGNs that harbor a dwarf Seyfert 1 nucleus; they are probably AGN-LINERs. Note, however, that none of these three galaxies are outstanding in the "inverted L" of Figure 2 in Paper I. As expected, weak AGNs can easily be masked by the contribution of the stellar population in a typical ground-based aperture observation. Hence, it could be that other strong-[O I] sources have less luminous AGNs than those in NGC 3998, NGC 4203, and NGC 4450 contributing to the optical continuum with less than $10 \%$ of the $4020 \AA$ light in a $0^{\prime \prime} 2 \times 1^{\prime \prime}$ aperture. NGC 2787 and NGC 4143 are two possible cases because they have a broad $\mathrm{H} \alpha$ emission component and have been classified by HFS97 as L1.9, but with a less luminous AGN because $x_{Y / \mathrm{FC}} \leq 10 \%$.

\subsection{Mean Age of the Stellar Population}

In Figure 13 we condense the results of the EPS analysis in the evolutionary diagram devised by Cid Fernandes et al. (2003). The trick consists of grouping the population vector onto just three components, which, because of normalization, are confined to a plane in this three-dimensional space, such that all components may be visualized in a two-dimensional projection. We choose to group the $10^{6}$ and $10^{7} \mathrm{yr}$ and powerlaw components in an $x_{Y / F C}$ component (as we did in the statistical discussion above) and plot it against the $10^{8} \mathrm{yr}$ fraction $\left(x_{8}\right)$. The two remaining components, $x_{9}$ and $x_{10}$, run along a third, perpendicular axis. The dotted lines in Figure 13 indicate lines of constant $x_{9+10} \equiv x_{9}+x_{10}$, as labeled. With these choices, young systems are located in the bottom right of this diagram, while $\sim 10^{8} \mathrm{yr}$ populations appear in the top left and systems dominated by populations of $10^{9} \mathrm{yr}$ or more occupy the bottom left corner. In other words, evolution proceeds counterclockwise in this handy diagnostic diagram of stellar populations (see Cid Fernandes et al. 2003 for details).

A first result that strikes the eye in this plot is the neat correspondence between the EPS results and the empirical stellar population classification. All but one of the $\eta=I$ objects are located above the $x_{9+10}=90 \%$ line, whereas most of the $\eta=O$ sources fall bellow this limit, with $\eta=I / O$ objects roughly in between. This correspondence, previously discussed in our statistical considerations ( $(4.1)$, is simply the EPS version of the excellent relationship between $\eta$ and the input spectral indices.

The distribution of LLAGNs in Figure 13 is suggestive of an evolutionary sequence, similar to that outlined in Paper I to explain the $[\mathrm{O} \mathrm{I}] / \mathrm{H} \alpha$ versus $W_{\mathrm{K}}$ diagram. In order to investi-

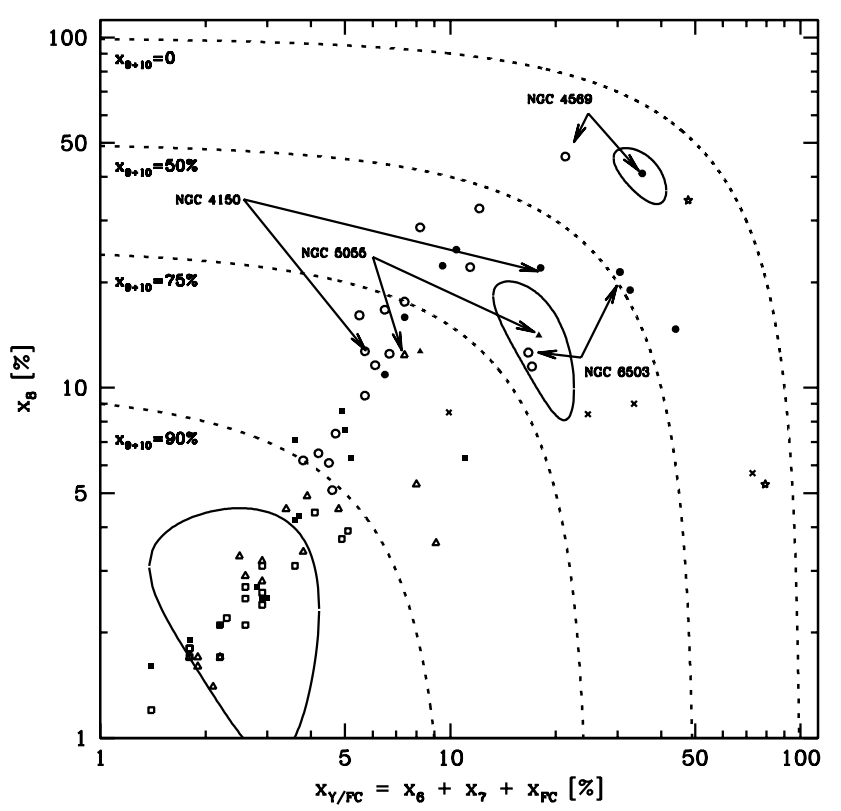

FIG. 13.-Two-dimensional projection of the EPS results. The young plus FC component is in the horizontal axis and the $10^{8} \mathrm{yr}$ stellar component in the vertical axis. A third perpendicular axis carries the remaining fraction of the flux, which goes in stars of $10^{9} \mathrm{yr}$ or more. Dotted lines represent the loci of constant $x_{9}+x_{10}$. Different symbols represent different $\eta$ classes of LLAGNs, as in Fig. 9. Arrows mark the position of several LLAGNs that have been observed from the ground (open symbols) and with STIS (filled symbols), and they show the variation of the stellar population due to the aperture effect. Ellipses show the uncertainties associated with three representative points: $\mathrm{NGC} 4569\left(x_{Y / \mathrm{FC}}=35 \% \pm 6 \%, x_{8}=41 \% \pm 7 \%\right)$, NGC $5055\left(x_{Y / \mathrm{FC}}=\right.$ $\left.18 \% \pm 5 \%, x_{8}=14 \% \pm 6 \%\right)$, and $\mathrm{NGC} 7331\left(x_{Y / \mathrm{FC}}=3 \% \pm 1 \%, x_{8}=\right.$ $3 \% \pm 2 \%$ ). Error ellipses have been computed using the errors and their covariances according to the expressions given by http://aeff.inta.es/users/ $\mathrm{mcs} / \mathrm{SED} /$ Theory/slide8.html. [See the electronic edition of the Journal for a color version of this figure.]

gate evolutionary effects in more detail, we have carried out an EPS analysis of theoretical galaxy spectra computed with the GISSEL96 evolutionary synthesis code of Bruzual \& Charlot (1993), processed through our EPS machinery in exactly the same manner as for the LLAGN data. Details of this "calibration" of EPS results in terms of evolutionary synthesis models are described in Cid Fernandes et al. (2003), where, except for the inclusion of a power law in the base, these same calculations were first discussed. Models were computed for a pure instantaneous burst (IB) with ages from 0 to 15 Gyr and for mixtures of an evolving IB and a fixed $15 \mathrm{Gyr}$ population. These mixed models are parameterized by the contrast parameter $c_{0}$, the fraction of the $4020 \AA$ flux that is associated with the $15 \mathrm{Gyr}$ old population when the burst starts $(t=0)$. These simplistic scenarios for the star formation history provide a useful reference for a qualitative evaluation of evolutionary effects.

In Figure 14 (left) we overlay these models with the LLAGN data in our evolutionary diagram. The outer solid line traces the evolution of a pure IB. The ages (in Myr) of some of the 21 models computed are indicated. Other solid lines in this plot correspond to mixed models with contrast parameters $c_{0}=40 \%, 60 \%$, and $80 \%$ (right to left). Notice that the evolutionary path of mixed models is similar in shape to that of a pure IB, but with much reduced amplitudes of the $10^{8} \mathrm{yr}$ and younger components. After $\sim 0.5 \mathrm{Gyr}$ all models merge in the sequence toward large $x_{9+10}$ in the bottom left of the plot. 


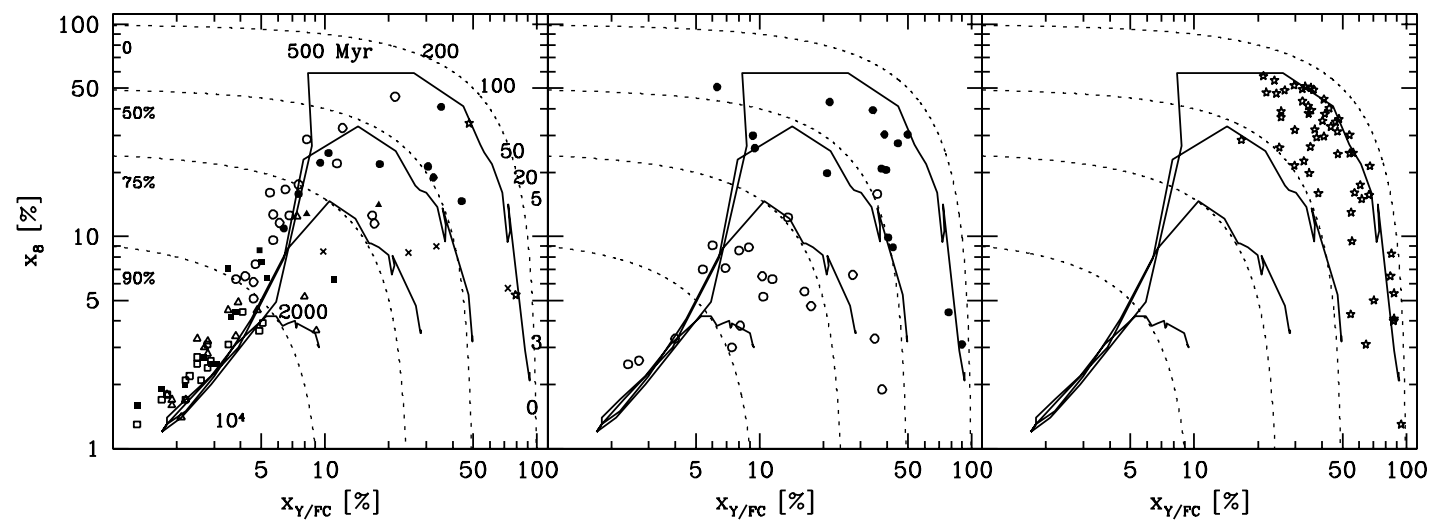

FIG. 14. - Comparison of the EPS results of LLAGNs (left), Seyfert 2 galaxies (middle), and starburst galaxies (right) in our "evolutionary diagram." Dotted lines in all panels mark contour constant $x_{9+10}$, as labeled in the left panel. Symbols in the left panel are as in Fig. 13. Solid lines trace the evolution of an IB in this EPS diagram. Labels along the outer evolutionary line in the left panel indicate the age (in Myr). The four different lines correspond to different contrast ratios $c_{O}$ of the burst to an underlying $15 \mathrm{Gyr}$ old population. The outer line corresponds to $c_{O}=0 \%$, i.e., a pure burst, while the inner lines correspond to $c_{0}=40 \%, 60 \%$, and $80 \%$ (right to left). These fractions refer to the time when the burst starts. [See the electronic edition of the Journal for a color version of this figure.]

The location of the LLAGNs in the above diagram reinforces our hypothesis that LLAGNs line up in what looks to be an evolutionary sequence, from $\eta=I$ to $O$. A similar conclusion can be reached comparing the location of these sources in the $D_{n}(4000)$ versus $\mathrm{H} \delta_{A}$ diagram with those of the models of Kauffmann et al. (2003). The transition from $\eta=I$ to $O$ occurs at 1-2 Gyr, depending on $c_{O}$. From the discussion in $\S 5.1$, this is also an evolutionary sequence in emission-line properties, from $\eta=I$ and weak [O $\mathrm{I}]$ to $\eta=O$ and either weak or strong $\left[\mathrm{O}_{\mathrm{I}}\right]$.

\subsection{Comparison with Seyfert 2 Galaxies: The Role of Starbursts in LLAGNS}

Our previous investigations of the stellar populations in type 2 Seyfert galaxies provide an interesting reference for comparison. Powerful circumnuclear starbursts have been unambiguously identified in $\sim 40 \%$ of nearby Seyfert 2 galaxies (Heckman et al. 1997; González Delgado et al. 1998, 2001; Storchi-Bergmann, Cid Fernandes, \& Schmitt 1998; Joguet et al. 2001). These starbursts were originally detected by means of either UV imaging, UV spectroscopy, or the W-R bump. Among other galaxy properties, these starbursts affect the equivalent width of the $\mathrm{Ca}$ II $\mathrm{K}$ line, which becomes diluted to $W_{\mathrm{K}}<10 \AA$ (Cid Fernandes et al. 2001a). Emissionline ratios are also affected. While in "pure Seyfert 2 galaxies" (defined as nuclei with no clear signs of starburst activity) line ratios like $\mathrm{He}{ }_{\mathrm{II}} / \mathrm{H} \beta$ and $[\mathrm{O} \mathrm{III}] / \mathrm{H} \beta$ assume a wide range of values, composite systems have systematically lower values of these ratios. The interpretation is straightforward. Since photoionization by a starburst contributes more to $\mathrm{H} \beta$ than to typical Seyfert 2 lines like He II $\lambda 4686$ and [O III] $\lambda 5007$, the presence of a starburst around a Seyfert 2 nucleus has the effect of diluting $\mathrm{He}{ }_{\text {III }} / \mathrm{H} \beta$ and $\left[\mathrm{O}{ }_{\mathrm{III}}\right] / \mathrm{H} \beta$ with respect to the pure AGN values. The resulting line ratios are in between those of starbursts and pure Seyfert 2 galaxies. In Cid Fernandes et al. (2001a) and Storchi-Bergmann et al. (2001) we further speculated that composite starburst plus Seyfert 2 systems evolve into pure Seyfert 2 galaxies.

The analogies with the results reported in this paper are evident. Our stellar LLAGNs, i.e., LLAGNs with HOBLs, diluted metal absorption lines, and low $[\mathrm{O} \mathrm{I}] / \mathrm{H} \alpha$, qualitatively resemble starburst plus Seyfert 2 composites. Similarly, LLAGNs with predominantly old stellar populations are analogous to pure Seyfert 2 galaxies. In broad terms, the results reported in this paper sound like a low-luminosity extension of the starburst-AGN connection identified in Seyfert 2 galaxies.

These similarities suggest that LLAGNs with HOBLs harbor a starburst. But do they? Undoubtedly, the most remarkable result of our survey is that HOBLs are ubiquitous, if not omnipresent, in weak-[O I] LLAGNs. The problem is that HOBLs are not direct signposts of ongoing star formation. Instead, they signal the presence of $10^{8}-10^{9} \mathrm{yr}$ populations (González Delgado et al. 2001). It is useful to recall here that for Seyfert 2 galaxies we have found that in all cases where young stars have been conclusively detected by UV data (Heckman et al. 1997; González Delgado et al. 1998), HOBLs are present in the optical. In other words, every time a young $\left(<10^{7} \mathrm{yr}\right)$ starburst is seen in the UV, a $\gtrsim 10^{8} \mathrm{yr}$ population is identified in the optical by its deep HOBLs, which points to multiple bursts or a roughly continuous star formation regime. Inverting and extrapolating from this result, the ubiquity of HOBLs in weak-[O I] LLAGNs could thus be interpreted as indirect evidence for the existence of young stars, which, as pointed out above, naturally explains the distribution of points in our $\left[\mathrm{O}_{\mathrm{I}}\right] / \mathrm{H} \alpha$ versus $W_{\lambda}$ and $x_{O}$ diagrams. However, when young starbursts are present in LLAGNs, they are generally faint (see Tables 3 and 4). The fact that LLAGNs are associated with low-luminosity starbursts may be a trivial consequence of a "starburst $\propto$ AGN" scaling law, which might exist for the mere reason that both starbursts and supermassive BHs feed on gas. As pointed out by Ho, Filippenko, \& Sargent (2003), the gaseous content of LINERs and TOs is small compared to that of Seyfert galaxies, which can potentially explain why starbursts are more easily found in Seyfert 2 galaxies than in LLAGNs.

Further insight can be gained by comparing the EPS of LLAGNs to results obtained in previous investigations. In Figure $14 b$ we plot the EPS of 35 Seyfert 2 galaxies from Cid Fernandes et al. (2001a; renormalized to $\lambda=4020 \AA$ ). Similarly, in Figure $14 c$ we plot the results for 57 starburst and $\mathrm{H}$ II galaxies from Cid Fernandes et al. (2003). Filled and open symbols in Figure $14 b$ represent, respectively, starburst plus Seyfert 2 composite systems and pure Seyfert 2 galaxies. Pure Seyfert 2 galaxies are less luminous than composites and comprise both objects dominated by old stars and sources that 
present an excess blue continuum whose nature we cannot ascertain with confidence (Storchi-Bergmann et al. 2000). The most likely sources for this blue continuum are scattered light from the AGNs and a relatively weak starburst.

Two differences stand out when comparing LLAGNs with Seyfert 2 galaxies. First, young starbursts appear far more frequently in Seyfert 2 galaxies. For instance, $\sim 40 \%$ of Seyfert 2 galaxies but less than $10 \%$ of the LLAGNs are located above the $x_{9+10}=50 \%$ line. ${ }^{11}$ In fact, most of the starburst plus Seyfert 2 composites populate the same region as bona fide starburst galaxies (Fig. 14c). Further evidence that young stars are more easily detected in Seyfert 2 galaxies than in LLAGNs is that whereas the W-R bump has been detected in $\sim 10 \%$ of the Seyfert 2 galaxies in the samples of Cid Fernandes et al. (2001a) and Joguet et al. (2001), we have not detected this feature in any of our LLAGNs. This does not imply that LLAGNs lack young massive stars altogether. After all, we know from our analysis and UV work (Maoz et al. 1998; Colina et al. 2002) that at least some LLAGNs contain these stars. Their relative contribution to the optical flux, however, is clearly much less relevant than in Seyfert 2 galaxies. Given the similarities in the host galaxy properties of Seyfert galaxies, LINERs, and TOs, this result implies that starbursts around LLAGNs are generally weaker than in Seyfert 2 galaxies.

Secondly, Seyfert 2 galaxies are visibly more spread out than LLAGNs in our evolutionary diagram. Interpreted in terms of stellar populations alone (i.e., neglecting the effects of scattered light), this result implies that Seyfert 2 galaxies have more mixed stellar populations as a result of stronger and/or more numerous starbursts in the recent $\left(\sim 10^{8} \mathrm{yr}\right)$ past. The strong clustering of LLAGNs along the evolutionary tracks for ages $\gtrsim 1$ Gyr is simply not seen for Seyfert 2 galaxies.

\subsection{Speculations on an Evolutionary Scenario}

It is further tempting to speculate that at least some Seyfert 2 galaxies evolve into LLAGNs. The intense star formation in Seyfert 2 composites must eventually cease with the continuous consumption of the gas supply. Barring further fueling events (e.g., interactions), passive evolution of the stellar populations would move these Seyfert 2 galaxies roughly along evolutionary paths between the pure IB and $c_{O}=40 \%$ models in Figure $14 a$, which leads to LLAGNs. If star formation gradually decays on timescales of $\sim 10^{8} \mathrm{yr}$ (instead of ceasing abruptly), after $\sim 10^{8} \mathrm{yr}$ a strong poststarburst (with pronounced HOBLs) would coexist with an incipient young population. This is the basic stellar mixture found in the TOs above the $c_{O}<40 \%$ track in Figure $14 a$. Concomitantly, the UV-blue continuum and emission-line luminosities of the system would decrease steadily because of the drop in star formation and accretion rates as gas becomes less and less available. Luminosities in the red should change less because of the steady output from the underlying old bulge population. At $\sim 1$ Gyr these systems would reach the "turnover" at $x_{Y / \mathrm{FC}} \sim 10$ and $x_{8} \sim 30 \%$ in Figure 14 . HOBLs from $\sim 10^{9} \mathrm{yr}$ populations would be present. Young stars, if present, should contribute little to the optical continuum and thus be hard to

\footnotetext{
11 Note, however, that we cannot exclude here that this difference could be due to a circumnuclear starburst $(100-500 \mathrm{pc})$ in Seyfert 2 galaxies, instead of young stars in the central $100 \mathrm{pc}$.
}

detect. In another billion years or so they would reach the $x_{9+10} \gtrsim 90 \%$ zone occupied by $\eta=O$ sources. The gas excitation in this phase would presumably be dominated by the AGN, which is consistent with the fact that strong-[O I $]$ sources are exclusively found in this region. The relative durations of these phases would become progressively longer along the evolutionary sequence, which seems to be quantitatively consistent with the demographics of Seyfert 2 galaxies and LLAGNs.

One can start this evolution from other initial positions in Figure 14, although $c_{0} \gtrsim 40 \%$ is required to turn a composite Seyfert 2 into $\eta=I$ TOs (compare Figs. $14 a$ and $14 b$ ). The pure Seyfert 2 galaxies between $x_{Y / \mathrm{FC}}=10 \%$ and $50 \%$ in Figure $14 b$ could well skip the $\eta=I$ phase altogether and move directly to $\eta=I / O$ or $O$ LLAGNs. Young stars, if present, would play an irrelevant role in the gas all the way along this sequence. The change from high- to low-excitation emission-line ratios, i.e., from Seyfert 2 to LINER, would be attributed to changes in the accretion rate. In summary, Seyfert 2 galaxies contain more younger starbursts than LLAGNs.

\section{SUMMARY AND CONCLUSIONS}

This is the second paper in a series that deals with the study of the stellar populations in LLAGNs. The goal is to determine whether the stellar populations in the central $20-100 \mathrm{pc}$ of these galaxies are related to the ionization processes of the nuclear gas. For this goal, we have collected STIS optical (2900-5700 ̊) spectra of 28 LLAGNs plus four H II nuclei and one nonactive galaxy from the HST archive. These data are added to the ground-based spectra of the LLAGNs presented in Paper I. In total, we have analyzed a sample of 73 LLAGNs, which represents $45 \%$ of this type of object in the HFS97 catalog. In this second paper we have (1) described the sample, $(2)$ classified the STIS nuclear $\left(0.2 \times 1^{\prime \prime}\right)$ stellar population by comparing with normal galaxies, (3) measured the equivalent widths and colors indicative of the stellar populations, (4) performed stellar population synthesis (EPS) of the whole sample, and (5) correlated EPS results with the emission-line ratio $\left[\mathrm{O}_{\mathrm{I}}\right] / \mathrm{H} \alpha$. LLAGNs are divided according to their $\left[\begin{array}{ll}\mathrm{O} & \mathrm{I}\end{array}\right] / \mathrm{H} \alpha$ ratio into two subtypes: strong $\left[\begin{array}{ll}\mathrm{O} & \mathrm{I}\end{array}\right]$ $([\mathrm{O} \mathrm{I}] / \mathrm{H} \alpha \geq 0.25)$ and weak $\left[\mathrm{O}_{\mathrm{I}}\right]([\mathrm{O} \mathrm{I}] / \mathrm{H} \alpha \leq 0.25)$. The main results are summarized below:

1. Weak-[O I] LLAGNs present a higher contribution of the intermediate-age population than strong-[O I] LLAGNs. They also seem to have a younger stellar population on average.

2. The relation between $\left[\mathrm{O}_{\mathrm{I}}\right] / \mathrm{H} \alpha$ and the stellar population suggests a stellar origin for the ionization of weak-[O $\mathrm{O}$ I LLAGNs with HOBLs in absorption, although the contribution of massive stars is small in the aperture of the ground-based spectra.

3. On the other hand, STIS spectra show a larger contribution of young stars in the case of weak-[O I] LLAGNs than in the ground-based data, suggesting that its reduced contribution is an effect of the lower contrast of the ground-based data. This result suggests that the dominant source in weak$\left[\begin{array}{ll}\mathrm{O} & \mathrm{I}\end{array}\right]$ LLAGNs with an intermediate-age population is thus stellar. This is confirmed by the HST UV spectra available for a few objects.

4. On the other hand, the dominant source of ionization in strong-[O I] LLAGNs is likely an AGN, which is consistent with the detection of broad Balmer lines in emission in a few 
cases and a larger contribution of older stars to their stellar population.

5. Evolutionary models for the stellar population suggest an age sequence in which the weak-[O I] LLAGNs are younger objects than the strong-[O I] LLAGNs. The evolution proceeds as the small starburst in the nuclei of the weak-[O I] LLAGNs fades away, decreasing the stellar contribution to the intensity of $\mathrm{H} \alpha$ and leading to a larger $\left[\mathrm{O}_{\mathrm{I}}\right] / \mathrm{H} \alpha$ ratio.

6. The evolutionary scenario proposed here is similar to the one we have previously proposed for Seyfert 2 galaxies (Storchi-Bergmann et al. 2001; Cid Fernandes et al. 2001a), in which a Seyfert 2 galaxy with a circumnuclear starburst (composite Seyfert 2) evolves to a "pure" Seyfert 2 galaxy as the starburst fades. The weak-[O I] LLAGNs seem to be lower luminosity counterparts of composite Seyfert 2 galaxies, while the strong-[O I] LLAGNs would be the pure LINERs. The difference between the Seyfert 2 galaxies and LINERs would be mainly due to the smaller accretion rate or efficiency in LINERs.
We thank Grazyna Stasinska, Miguel Cerviño, and Luis Colina for their helpful comments from a thorough reading of the paper and the referee for her/his suggestions that helped to improve the paper. R. G. D. and E. P. acknowledge support by the Spanish Ministry of Science and Technology (MCyT) through grants AYA-2001-3939-C03-01 and AYA-2001-2089. R. C. F. and T. S.-B. acknowledge support from $\mathrm{CNPq}$ and CAPES. Some of these data are from observations made with the NASA/ESA Hubble Space Telescope, obtained from the data archive at the ST-ECF. Additional data presented here have been taken using ALFOSC, which is owned by the Instituto de Astrofísica de Andalucía (IAA) and operated at the Nordic Optical Telescope under agreement between IAA and the NBIfAFG of the Astronomical Observatory of Copenhagen. We are grateful to the IAA director for the allocation of 5.5 nights of the ALFOSC guaranteed time for this project. The National Radio Astronomy Observatory is a facility of the National Science Foundation, operated under cooperative agreement by Associated Universities, Inc.

\section{REFERENCES}

Balogh, M. L., Morris, S. L., Yee, H. K. C., Carlberg, R. G., \& Ellingson, E. 1999, ApJ, 527, 54

Barth, A., \& Shields, J. C. 2000, PASP, 112, 753

Bica, E., \& Alloin, D. 1986a, A\&A, 162, 21 1986b, A\&AS, 66, 171

Binette, L., Magris, C. G., Stasinska, G., \& Bruzual, A. G. 1994, A\&A, 292, 13 Bruzual, G., \& Charlot, S. 1993, ApJ, 405, 538

Cid Fernandes, R., Heckman, T., Schmitt, H., González Delgado, R. M., \& Storchi-Bergmann, T. 2001a, ApJ, 558, 81

Cid Fernandes, R., Leão, J. R. S., \& Rodrigues-Lacerda, R. 2003, MNRAS, 340,29

Cid Fernandes, R., Sodré, L., Schmitt, H., \& Leão, J. R. S. 2001b, MNRAS, 325,60

Cid Fernandes, R., \& Terlevich, R. 1995, MNRAS, 272, 423

Cid Fernandes, R., et al. 2004, ApJ, 605, 105 (Paper I)

Colina, L., González Delgado, R. M., Mas-Hesse, J. M., Leitherer, C. \& Jiménez-Bailón, E. 2002, ApJ, 579, 545

Filippenko, A. V. 1996, in ASP Conf. Ser. 103, The Physics of LINERs in View of Recent Observations, ed. M. Eracleous, A. Koratkar, C. Leitherer, \& L. Ho (San Francisco: ASP), 17

Filippenko, A. V., \& Terlevich, R. 1992, ApJ, 397, L79

González Delgado, R. M., Heckman, T., \& Leitherer, C. 2001, ApJ, 546, 845

González Delgado, R. M., Heckman, T., Leitherer, C., Meurer, G., Krolik, J., Wilson, A. S., Kinney, A., \& Koratkar, A. 1998, ApJ, 505, 174

González Delgado, R. M., Leitherer, C., \& Heckman, T. M. 1999, ApJS, 125,489

González Delgado, R. M., et al. 2003, in ASP Conf. Ser. 297, Star Formation through Time, ed. E. Perez, R. M. González Delgado, \& G. Tenorio-Tagle (San Francisco: ASP), 417

Heckman, T. M., González Delgado, R. M., Leitherer, C., Meurer, G., Krolik, J., Kinney, A., Koratkar, A., \& Wilson, A. S. 1997, ApJ, 482, 114

Ho, L. C., Filippenko, A. V., \& Sargent, W. L. W. 1997a, ApJS, 112, 315 (HFS97)

1997b, ApJS, 112, 391

2003, ApJ, 583, 159
Ho, L. C., Rudnick, G., Rix, H.-W., Shields, J. C., McIntosh, D. H., Filippenko, A. V., Sargent, W. L. W., \& Eracleous, M. 2000, ApJ, 541, 120

Ho, L. C., et al. 2001, ApJ, 549, L51

Jiménez-Bailón, E., Santos-Lleó, M., Mas-Hesse, J. M., Guainazzi, M., Colina,

L., Cerviño, M., \& González Delgado, R. M. 2003, ApJ, 593, 127

Joguet, B., Kunth, D., Melnick, J., Terlevich, R., \& Terlevich, E. 2001, A\&A, 380,19

Kauffmann, G., et al. 2003, MNRAS, 341, 54

Maoz, D., Koratkar, A., Shields, J. C., Ho, L. C., Filippenko, A. V., \& Sternberg, A. 1998, AJ, 116, 55

Pogge, R. W., \& Martini, P. 2002, ApJ, 569, 624

Raimann, D., Storchi-Bergmann, T., González Delgado, R. M., Cid Fernandes, R., Heckman, T., Leitherer, C., \& Schmitt, H. 2003, MNRAS, 339, 772

Schmidt, A. A., Copetti, M. V. F., Alloin, D., \& Jablonka, P. 1991, MNRAS, 249, 766

Schmitt, H. R., Storchi-Bergmann, T., \& Cid Fernandes, R. 1999, MNRAS, 303, 173

Shields, J. C. 1992, ApJ, 399, L27

Shields, J. C., Rix, H.-W., McIntosh, D. H., Ho, L. C., Rudnick, G., Filippenko, A. V., Sargent, W. L. W., \& Sarzi, M. 2000, ApJ, 534, L27

Smith, L. J., Norris, R. P. F., \& Crowther, P. A. 2002, MNRAS, 337, 1309

Stasinska, G. 1984, A\&A, 135, 341

Storchi-Bergmann, T., Cid Fernandes, R., \& Schmitt, H. R. 1998, ApJ, 501, 94

Storchi-Bergmann, T., Eracleous, M., Ruiz, M. T., Livio, M., Wilson, A. S., \& Filippenko, A. V. 1997, ApJ, 489, 87

Storchi-Bergmann, T., González Delgado, R. M., Schmitt, H. R., Cid Fernandes, R., \& Heckman, T. 2001, ApJ, 559, 147

Storchi-Bergmann, T., Raimann, D., Bica, E. L. D., \& Fraquelli, H. A. 2000, ApJ, 544, 747

Taniguchi, Y., Shioya, Y., \& Murayama, T. 2000, AJ, 120, 1265

Terashima, Y., Ho, L. C., \& Ptak, A. F. 2000, ApJ, 539, 161

Terlevich, R., \& Melnick, J. 1985, MNRAS, 213, 841

Worthey, G., \& Ottaviani, D. L. 1997, ApJS, 111, 377 